\title{
On fractional integro-differential inclusions via the extended fractional Caputo-Fabrizio derivation
}

\author{
Dumitru Baleanu ${ }^{1,2}$, Shahram Rezapour ${ }^{3^{*}}$ and Zohreh Saberpour ${ }^{3}$
}

\section{"Correspondence:}

rezapourshahram@yahoo.ca; sh.rezapour@azaruniv.ac.ir

${ }^{3}$ Department of Mathematics,

Azarbaijan Shahid Madani

University, Tabriz, Iran

Full list of author information is

available at the end of the article

\begin{abstract}
We first show that four fractional integro-differential inclusions have solutions. Also, we show that dimension of the set of solutions for the second fractional integro-differential inclusion problem is infinite dimensional under some different conditions.
\end{abstract}

MSC: Primary 34A08; secondary 34A60

Keywords: Caputo-Fabrizio fractional derivation; Dimension of the set of solutions; Fractional differential inclusion

\section{Introduction}

A lot of papers on fractional differential equations (see, for example, [1-18] and the references therein) have been published. As you know, most famous fractional derivations are the Caputo and Riemann-Liouville derivations. In 2015, Caputo and Fabrizio introduced a new fractional derivation without singular kernel [19]. Some researchers published some works about solving different equations including the new derivation (see, for example, $[2,3,10,20-25])$. Some researchers investigated some results on dimension of the set of solutions for some fractional differential inclusions (see, for example, [26]).

Let $b>0, u \in H^{1}(0, b)$, and $\zeta \in(0,1)$. As you know, the Caputo-Fabrizio fractional derivative of order $\zeta$ is defined by

$$
{ }^{\mathrm{CF}} \mathcal{D}^{\zeta} u(t)=\frac{(2-\zeta) M(\zeta)}{2(1-\zeta)} \int_{0}^{t} \exp \left(\frac{-\zeta}{1-\zeta}(t-s)\right) u^{\prime}(s) d s
$$

where $t \geq 0$ and $M(\zeta)$ is a normalization constant depending on $\zeta$ such that $M(0)=$ $M(1)=1$ [19]. Losada and Nieto showed that ${ }^{\mathrm{CF}} \mathcal{I}^{\zeta} u(t)=\frac{2(1-\zeta)}{(2-\zeta) M(\zeta)} u(t)+\frac{\zeta}{(2-\zeta) M(\zeta)} \int_{0}^{t} u(s) d s$ [27]. Also, they showed that $M(\zeta)=\frac{2}{2-\zeta}$ [27]. Hence, the fractional Caputo-Fabrizio derivative of order $\zeta$ is given by ${ }^{\mathrm{CF}} \mathcal{D}^{\zeta} u(t)=\frac{1}{1-\zeta} \int_{0}^{t} \exp \left(-\frac{\zeta}{1-\zeta}(t-s)\right) u^{\prime}(s) d s$, when $t \geq 0$ and $0<\zeta<1$ [27]. If $n \geq 1$ and $\zeta \in(0,1)$, then the fractional derivative ${ }^{\mathrm{CF}} \mathcal{D}^{\zeta+n}$ of order $n+\zeta$ is defined by ${ }^{\mathrm{CF}} \mathcal{D}^{\zeta+n} u:={ }^{\mathrm{CF}^{\zeta}} \mathcal{D}^{\zeta}\left(\mathcal{D}^{n} u(t)\right)$ [27]. Let $u, v \in H^{1}(0,1)$ and $\zeta \in(0,1)$. If $u^{(s)}(0)=0$ for all $s=1,2, \ldots, n$, then ${ }^{\mathrm{CF}} \mathcal{D}^{\zeta}\left({ }^{\mathrm{CF}} \mathcal{D}^{n}(u(t))={ }^{\mathrm{CF}} \mathcal{D}^{n}\left({ }^{\mathrm{CF}} \mathcal{D}^{\zeta}(u(t))\right.\right.$. Also, we have $\lim _{\zeta \rightarrow 0}{ }^{\mathrm{CF}} \mathcal{D}^{\zeta} u(t)=u(t)-u(0), \lim _{\zeta \rightarrow 1}{ }^{\mathrm{CF}} \mathcal{D}^{\zeta} u(t)=u(t)^{\prime}$, and ${ }^{\mathrm{CF}} \mathcal{D}^{\zeta}(\lambda u(t)+\gamma v(t))=$ (http://creativecommons.org/licenses/by/4.0/), which permits unrestricted use, distribution, and reproduction in any medium, provided you give appropriate credit to the original author(s) and the source, provide a link to the Creative Commons license, and indicate if changes were made. 
$\lambda^{\mathrm{CF}} \mathcal{D}^{\zeta} u(t)+\gamma^{\mathrm{CF}} \mathcal{D}^{\zeta} v(t)$ [27]. It has been proved that the unique solution for the problem ${ }^{\mathrm{CF}} \mathcal{D}^{\zeta} u(t)=v(t)$ with boundary condition $u(0)=c$ is given by $u(t)=c+a_{\zeta}(v(t)-v(0))+$ $b_{\zeta} \int_{0}^{t} v(s) d s$, where $a_{\zeta}=\frac{2(1-\zeta)}{(2-\zeta) M(\zeta)}=1-\zeta$ and $b_{\zeta}=\frac{2 \zeta}{(2-\zeta) M(\zeta)}=\zeta$ ([19] and [27]). Note that $v(0)=0$. Suppose that $u, v \in C_{\mathbb{R}}[0,1], u(0)=0$, and there is a real constant $L$ such that $|u(t)-v(t)| \leq L$ for all $t \in[0,1]$. Recently, Baleanu, Mousalou, and Rezapour proved that $\left|{ }^{\mathrm{CF}} \mathcal{D}^{\zeta} u(t)-{ }^{\mathrm{CF}} \mathcal{D}^{\zeta} v(t)\right| \leq \frac{1}{(1-\zeta)^{2}} L$ for all $t \in[0,1]$ [10]. This leads to $\left|{ }^{\mathrm{CF}} \mathcal{D}^{\zeta} u(t)\right| \leq\left(\frac{1}{(1-\zeta)^{2}}\right) L$ for all $t \in[0,1]$ whenever $u \in C_{\mathbb{R}}[0,1]$ and $|u(t)| \leq L$ for some $L \geq 0$ and all $t \in[0,1]$ with $u(0)=0$ [10]. Also, they showed that $\left|{ }^{\mathrm{CF}} \mathcal{I}^{\zeta} u(t)-{ }^{\mathrm{CF}} \mathcal{I}^{\zeta} v(t)\right| \leq L$ for all $t \in[0,1]$ [10] and so $\left|{ }^{\mathrm{CF}} \mathcal{I}^{\zeta} u(t)\right| \leq L$ for all $t \in[0,1]$ whenever $u \in C_{\mathbb{R}}[0,1]$ with $|u(t)| \leq L$ for some $L \geq 0$ and all $t \in[0,1]$. For some more necessary definitions, see [1].

Let $u \in C_{\mathbb{R}}[0, d], d>0$ and $\zeta \in(0,1)$. The extended fractional Caputo-Fabrizio derivation of order $\zeta$ is defined by [11]

$$
\begin{aligned}
{ }_{N}^{\mathrm{CF}} \mathcal{D}^{\zeta} u(t)= & \frac{B(\zeta)}{1-\zeta}(u(t)-u(0)) \exp \left(\frac{-\zeta}{1-\zeta} t\right) \\
& +\frac{\zeta B(\zeta)}{(1-\zeta)^{2}} \int_{0}^{t}(u(t)-u(s)) \exp \left(\frac{-\zeta}{1-\zeta}(t-s)\right) d s
\end{aligned}
$$

If $u(0)=0$, then we have ${ }_{N}^{\mathrm{CF}} \mathcal{D}^{\zeta} u(t)=\frac{B(\zeta)}{1-\zeta} u(t)-\frac{\zeta B(\zeta)}{(1-\zeta)^{2}} \int_{0}^{t} \exp \left(-\frac{\zeta}{1-\zeta}(t-s)\right) u(s) d s[11]$

Lemma 1 ([11]) Let $u \in H^{1}(0, b), b>0$, and $\zeta \in(0,1)$. Then ${ }_{N}{ }^{\mathrm{F}} \mathcal{D}^{\zeta} u(t)={ }^{\mathrm{CF}} \mathcal{D}^{\zeta} u(t)$. If $u \in$ $C_{\mathbb{R}}[0, b]$, then $\lim _{\zeta \rightarrow 0}{ }_{N}^{C F} \mathcal{D}^{\zeta} u(t)=u(t)-u(0)$.

Lemma 2 ([11]) Let $0<\zeta<1$. Then a solution for the problem ${ }_{N}^{\mathrm{CF}} \mathcal{D}^{\zeta} u(t)=v(t)$ with boundary condition $u(0)=0$ is given by $u(t)=a_{\zeta} v(t)+b_{\zeta} \int_{0}^{t} v(s) d s$.

Lemma 3 ([11]) Let $u, v \in C_{\mathbb{R}}[0,1]$. If there is a real constant $L$ such that $|u(t)-v(t)| \leq L$ for all $t \in[0,1]$, then $\left.\right|_{N} ^{\mathrm{CF}} \mathcal{D}^{\zeta} u(t)-{ }_{N}^{\mathrm{CF}} \mathcal{D}^{\zeta} v(t) \mid \leq \frac{(2-\zeta) B(\zeta)}{(1-\zeta)^{2}} L$ for all $t \in[0,1]$. If $u(0)=v(0)$, then $\left.\right|_{N} ^{\mathrm{CF}} \mathcal{D}^{\zeta} u(t)-{ }_{N}^{\mathrm{CF}} \mathcal{D}^{\zeta} v(t) \mid \leq \frac{B(\zeta)}{(1-\zeta)^{2}} L$.

This result implies that $\left.\right|_{N} ^{\mathrm{CF}} \mathcal{D}^{\zeta} u(t) \mid \leq \frac{(2-\zeta) B(\zeta)}{(1-\zeta)^{2}} L$ for all $t \in[0,1]$ whenever $u \in C_{\mathbb{R}}[0,1]$ with $|u(t)| \leq L$ for some $L \geq 0$ and all $t \in[0,1]$.

We need the following results.

Lemma 4 ([28]) Suppose that $\mathcal{Y}$ is a Banach space, $\mathcal{F}: I \times \mathcal{Y} \rightarrow \mathcal{P}_{c p, c v}(\mathcal{Y})$ is an $L^{1}$ Caratheodory multivalued and $\epsilon$ is a linear continuous mapping from $L^{1}(I, \mathcal{Y})$ to $C(I, \mathcal{Y})$. Then the mapping $\epsilon \circ S_{\mathcal{F}}: C(I, \mathcal{Y}) \rightarrow \mathcal{P}_{c p, c v} C(I, \mathcal{Y})$ defined by $\left(\epsilon \circ S_{\mathcal{F}}\right)(y)=\epsilon\left(S_{\mathcal{F}, y}\right)$ is a closed graph mapping in $C(I, \mathcal{Y}) \times C(I, \mathcal{Y})$.

Theorem 5 ([29]) Assume that $Y$ is a Banach space, $D$ is a closed and convex subset of $Y$, and $W$ is an open subset of $D$ with $0 \in W$.If $\mathcal{F}: \bar{W} \rightarrow P_{c p, c}(D)$ is an upper semi-continuous compact map, then either $\mathcal{F}$ has a fixed point in $\bar{W}$ or there is $x \in \partial W$ and $\delta \in(0,1)$ such that $x \in \delta \mathcal{F}(x)$.

Theorem 6 ([30]) Suppose that $(\mathcal{Y}, d)$ is a complete metric space. If $\mathcal{G}: \mathcal{Y} \rightarrow P_{c l}(\mathcal{Y})$ is a contraction, then $\mathcal{G}$ has a fixed point. 
Theorem 7 ([31]) Assume that $\mathcal{Y}$ is a Banach space, $\mathcal{E} \in P_{b d, c l, c v}(\mathcal{Y})$ and $\mathcal{F}, \mathcal{G}: \mathcal{E} \rightarrow$ $P_{c p, c v}(\mathcal{Y})$ are two multivalued operators. If $\mathcal{F} y+\mathcal{G} y \subset \mathcal{E}$ for all $y \in \mathcal{E}, \mathcal{F}$ is a contraction and $\mathcal{G}$ is an upper semi-continuous compact map, then there is $y \in \mathcal{E}$ such that $y \in \mathcal{F} y+\mathcal{G} y$.

Theorem 8 ([32]) Assume that $\mathcal{Y}$ is a Banach algebra, $D \in \mathcal{P}_{b d, c l, c v}(\mathcal{Y})$ and $\mathcal{F}_{1}: D \rightarrow$ $\mathcal{P}_{c l, c v, b d}(\mathcal{Y})$ and $\mathcal{F}_{2}: D \rightarrow \mathcal{P}_{c p, c v}(\mathcal{Y})$ are two set-valued maps such that $\mathcal{F}_{1}$ is Lipschitz with a Lipschitz constant $\delta, \mathcal{F}_{2}$ is upper semi-continuous and compact, $\mathcal{F}_{1} x \mathcal{F}_{2} x$ is a convex subset $D$ for all $x \in D$ and $\mathcal{N} \delta<1$, where $\mathcal{N}=\left\|\mathcal{F}_{2}(D)\right\|=\sup \left\{\left\|\mathcal{F}_{2} x\right\|: x \in D\right\}$. Then there is $y \in D$ such that $y \in \mathcal{F}_{1} y \mathcal{F}_{2} y$.

Lemma 9 ([26]) Let $\mathcal{A}$ mapping $[0,1]$ into $\mathcal{P}_{c p, c v}(\mathbb{R})$ be measurable such that the Lebesgue measure of the set $\{t: \operatorname{dim} \mathcal{A}(t)<1\}$ is zero. Then there are arbitrarily many linearly independent measurable selections $y_{1}(\cdot), \ldots, y_{m}(\cdot)$ of $\mathcal{A}$.

Theorem 10 ([26]) Let $\mathcal{H}$ be a nonempty closed convex subset of a Banach space $\mathcal{Y}$ and $\mathcal{F}: \mathcal{H} \rightarrow \mathcal{P}_{c p, c v}(\mathcal{H})$ be a $\delta$-contraction. If $\operatorname{dim} \mathcal{F}(x) \geq m$ for all $x \in \mathcal{H}$, then $\operatorname{dim} F i x(\mathcal{F}) \geq m$.

\section{Main results}

Consider the Banach space $\mathcal{X}=C(I)$ of real-valued continuous functions on $I=[0,1]$ via the norm $\|x\|=\sup _{t \in I}|x(t)|$. Assume that $\zeta, \iota:[0,1] \times[0,1] \rightarrow[0, \infty)$ are two continuous maps such that $\sup \left|\int_{0}^{t} \iota(t, s) d s\right|<\infty$ and $\sup \left|\int_{0}^{t} \zeta(t, s) d s\right|<\infty$. Consider the maps $\phi$ and $\varphi$ defined by $(\phi w)(t)=\int_{0}^{t} \zeta(t, s) w(s) d s$ and $(\varphi w)(t)=\int_{0}^{t} \iota(t, s) w(s) d s$. Suppose that $\eta(t) \in$ $L^{\infty}(I)$ with $\eta^{*}=\sup _{t \in I}|\eta(t)|$. Put $\zeta_{0}=\sup \left|\int_{0}^{t} \zeta(t, s) d s\right|$ and $\iota_{0}=\sup \left|\int_{0}^{t} \iota(t, s) d s\right|$. First, we are going to investigate the fractional integro-differential inclusion

$$
{ }_{N}^{\mathrm{CF}} \mathcal{D}^{\zeta} x(t) \in \mathcal{F}\left(t, x(t),(\phi x)(t),(\varphi x)(t),{ }_{N}^{\mathrm{CF}} \mathcal{D}^{\beta_{1}} x(t),{ }_{N}^{\mathrm{CF}} \mathcal{D}^{\beta_{2}} x(t), \ldots,{ }_{N}^{\mathrm{CF}} \mathcal{D}^{\beta_{m}} x(t)\right),
$$

with boundary condition $x(0)=0$, where $\zeta, \beta_{1}, \ldots, \beta_{m} \in(0,1)$.

We say that a function $x \in \mathcal{X}$ is a solution for problem (1) whenever there exists a function $f \in C(I)$ such that

$$
f(t) \in \mathcal{F}\left(t, x(t),(\phi x)(t),(\varphi x)(t),{ }_{N}^{C F} \mathcal{D}^{\beta_{1}} x(t),{ }_{N}^{C F} \mathcal{D}^{\beta_{2}} x(t), \ldots,{ }_{N}^{C F} \mathcal{D}^{\beta_{m}} x(t)\right)
$$

for almost all $t \in I$ and $x(t)=a_{\zeta} f(t)+b_{\zeta} \int_{0}^{t} f(s) d s$.

Theorem 11 Let $\mathcal{F}: I \times \mathbb{R}^{m+3} \rightarrow P_{c p, c v}(\mathbb{R})$ be a Caratheodory multivalued map such that

$$
\begin{aligned}
\left\|\mathcal{F}\left(t, x_{1}, x_{2}, x_{3}, y_{1}, \ldots, y_{m}\right)\right\|_{p} & =\sup \left\{|y|: y \in \mathcal{F}\left(t, x_{1}, x_{2}, x_{3}, y_{1}, \ldots, y_{m}\right)\right\} \\
& \leq \eta(t)\left(\left|x_{1}\right|+\left|x_{2}\right|+\left|x_{3}\right|+\sum_{i=1}^{m}\left|y_{i}\right|\right)
\end{aligned}
$$

for all $t \in I, x_{i}, y_{j} \in \mathbb{R}, 1 \leq i \leq 3$ and $1 \leq j \leq m$. If $\eta^{*}\left(1+\zeta_{0}+\iota_{0}+\sum_{i=1}^{m} \frac{B\left(\beta_{i}\right)}{\left(1-\beta_{i}\right)^{2}}\right) \leq 1$, then inclusion (1) has one solution.

Proof For $x \in \mathcal{X}$, define a selection set of $\mathcal{F}$ at $x \in \mathcal{X}$ by

$$
\begin{aligned}
S_{\mathcal{F}, x}:= & \left\{f \in L^{1}(I, R): f(t) \in \mathcal{F}(t, x(t),(\phi x)(t),(\varphi x)(t),\right. \\
& \left.\left.{ }_{N}^{\mathrm{CF}} \mathcal{D}^{\beta_{1}} x(t),{ }_{N}^{\mathrm{CF}} \mathcal{D}^{\beta_{2}} x(t), \ldots,{ }_{N}{ }_{N} \mathcal{D}^{\beta_{m}} x(t)\right) \text { for all } t \in I\right\} .
\end{aligned}
$$


Since $\mathcal{F}$ is a Caratheodory multifunction, by using Theorem 1.3.5 in [33], we get $S_{\mathcal{F}, x}$ is nonempty. Define an operator $\Omega: \mathcal{X} \rightarrow P(\mathcal{X})$ by $\Omega(x)=\{g \in \mathcal{X}$ : there exists $f \in$ $S_{\mathcal{F}, x}$ such that $g(t)=a_{\zeta} f(t)+b_{\zeta} \int_{0}^{t} f(s) d s$ for all $\left.t \in I\right\}$. We show that the operator $\Omega$ satisfies the hypothesis of Theorem 5. First, we show that $\Omega(x)$ is convex for all $\in \mathcal{X}$.

Let $g_{1}, g_{2} \in \Omega(x)$ and $w \in[0,1]$. Choose $f_{1}, f_{2} \in S_{\mathcal{F}, x}$ such that $g_{i}(t)=a_{\zeta} f_{i}(t)+b_{\zeta} \int_{0}^{t} f_{i}(s) d s$ for all $t \in I$. Then we have

$$
\left[w g_{1}+(1-w) g_{2}\right](t)=a_{\zeta}\left(w f_{1}+(1-w) f_{2}\right)(t)+b_{\zeta} \int_{0}^{t}\left(w f_{1}+(1-w) f_{2}\right)(s) d s
$$

for all $t \in I$. Since $\mathcal{F}$ has convex values, it is easy to check that $S_{\mathcal{F}, x}$ is convex, and so $w g_{1}+(1-w) g_{2} \in \Omega(x)$. Now, we show that $\Omega$ maps bounded sets into bounded subsets. Let $\mathcal{B}_{r}=\{x \in \mathcal{X}:\|x\| \leq r\}, x \in \mathcal{B}_{r}$, and $g \in \Omega(x)$. Choose $f \in S_{\mathcal{F}, x}$ such that

$$
\begin{aligned}
|g(t)| \leq & a_{\zeta}|f(t)|+b_{\zeta} \int_{0}^{t}|f(s)| d s \leq a_{\zeta} \eta(t)(|x|+|\varphi(x)|+|\phi(x)| \\
& \left.+\left|{ }_{N}^{\mathrm{CF}} \mathcal{D}^{\beta_{1}} x(t)\right|+\left|{ }_{N}^{\mathrm{CF}} \mathcal{D}^{\beta_{2}} x(t)\right|+\cdots+\left|{ }_{N}^{\mathrm{CF}} \mathcal{D}^{\beta_{m}} x(t)\right|\right) \\
& +b_{\zeta} \int_{0}^{t}(|x|+|\varphi(x)|+|\phi(x)| \\
& \left.+\left|{ }_{N}^{\mathrm{CF}} \mathcal{D}^{\beta_{1}} x(s)\right|+\left|{ }_{N}^{\mathrm{CF}} \mathcal{D}^{\beta_{2}} x(s)\right|+\cdots+\left|{ }_{N}^{\mathrm{CF}} \mathcal{D}^{\beta_{m}} x(s)\right|\right) \eta(s) d s \\
\leq & a_{\zeta} \eta^{*}\left(r+\zeta_{0} r+\iota_{0} r+\sum_{i=1}^{m} \frac{B\left(\beta_{i}\right)}{\left(1-\beta_{i}\right)^{2}} r\right) \\
& +b_{\zeta} \eta^{*}\left(r+\zeta_{0} r+\iota_{0} r+\sum_{i=1}^{m} \frac{B\left(\beta_{i}\right)}{\left(1-\beta_{i}\right)^{2}} r\right) \\
= & \eta^{*} \cdot r \cdot\left(1+\zeta_{0}+\iota_{0}+\sum_{i=1}^{m} \frac{B\left(\beta_{i}\right)}{\left(1-\beta_{i}\right)^{2}}\right)\left(a_{\zeta}+b_{\zeta}\right) \leq r .
\end{aligned}
$$

Thus, $\|g\|=\max _{t \in I}|g(t)| \leq r$. This implies that $\Omega$ maps bounded sets into bounded sets in $\mathcal{X}$. Now, we show that $\Omega$ maps bounded sets of $\mathcal{X}$ into equi-continuous sets. Let $t_{1}, t_{2} \in I$ with $t_{1}<t_{2}, x \in \mathcal{B}_{r}$ and $g \in \Omega(x)$. Then we have

$$
\begin{aligned}
\left|g\left(t_{2}\right)-g\left(t_{1}\right)\right| & =\left|a_{\zeta} f\left(t_{2}\right)+b_{\zeta} \int_{0}^{t_{2}} f(s) d s-a_{\zeta} f\left(t_{1}\right)-b_{\zeta} \int_{0}^{t_{1}} f(s) d s\right| \\
& \leq a_{\zeta}\left|f\left(t_{2}\right)-f\left(t_{1}\right)\right|+b_{\zeta} \int_{t_{1}}^{t_{2}}|f(s)| d s \\
& \leq r\left(1+\zeta_{0}+\iota_{0}+\sum_{i=1}^{m} \frac{B\left(\beta_{i}\right)}{\left(1-\beta_{i}\right)^{2}}\right)\left(\eta\left(t_{2}\right)-\eta\left(t_{1}\right)\right)\left(a_{\zeta}+b_{\zeta}\right) .
\end{aligned}
$$

Hence, the right-hand side of the inequality tends to zero (independent on $x \in \mathcal{B}_{r}$ ) as $t_{2} \rightarrow t_{1}$. This implies that $\Omega: \mathcal{X} \rightarrow P(\mathcal{X})$ is a compact multivalued map by using the Arzela-Ascoli theorem. We show that $\Omega$ has a closed graph. Let $x_{n} \rightarrow x_{*}, g_{n} \in \Omega\left(x_{n}\right)$ for all $n$ and $g_{n} \rightarrow g_{*}$. It is sufficient to prove that $g_{*} \in \Omega\left(x_{*}\right)$. Since $g_{n} \in \Omega\left(x_{n}\right)$ for all $n$, there exist $f_{n} \in S_{\mathcal{F}, x_{n}}$ such that $g_{n}(t)=a_{\zeta} f_{n}(t)+b_{\zeta} \int_{0}^{t} f_{n}(s) d s$ for all $t \in I$. Thus, we have to show that there exist $f_{*} \in S_{\mathcal{F}, x_{*}}$ such that $g_{*}(t)=a_{\zeta} f_{*}(t)+b_{\zeta} \int_{0}^{t} f_{*}(s) d s$ for all $t \in I$. Consider 
the linear continuous operator $\theta: L^{1}(I, \mathbb{R}) \rightarrow \mathcal{X}$ defined by $f \mapsto \theta(f)(t)$, where $\theta(f)(t)=$ $a_{\zeta} f(t)+b_{\zeta} \int_{0}^{t} f(s) d s$ for all $t \in I$. Since $\theta$ is a linear continuous map, by using Lemma 4 we get $\theta \circ S_{\mathcal{F}}$ is a closed graph operator. Note that $g_{n} \in \theta \circ S_{\mathcal{F}}\left(x_{n}\right)$ for all $n$. Since $x_{n} \rightarrow x_{*}$ and $g_{n} \rightarrow g_{*}$, there exists $f_{*} \in S_{\mathcal{F}}\left(x_{*}\right)$ such that $g_{*}(t)=a_{\zeta} f_{*}(t)+b_{\zeta} \int_{0}^{t} f_{*}(s) d s$ for all $t \in I$. For $\lambda \in(0,1)$ and $x \in \lambda \Omega(x)$, there exists $f \in S_{\mathcal{F}, x}$ such that $x(t)=a_{\zeta} \lambda f(t)+b_{\zeta} \int_{0}^{t} \lambda f(s) d s$ for all $t \in I$. Hence,

$$
|x(t)| \leq \lambda\left(a_{\zeta}+b_{\zeta}\right) \eta^{*} \cdot\left(1+\zeta_{0}+\iota_{0}+\sum_{i=1}^{m} \frac{B\left(\beta_{i}\right)}{\left(1-\beta_{i}\right)^{2}}\right)\|x\| .
$$

Thus, $\|x\|=\max _{t \in I}|x(t)| \leq \lambda\|x\|$. Put $\mathcal{W}=\left\{x \in \mathcal{X},\|x\|<r\left(1+\zeta_{0}+\iota_{0}+\sum_{i=1}^{m} \frac{B\left(\beta_{i}\right)}{\left(1-\beta_{i}\right)^{2}}\right)\right\}$. Note that the operator $\Omega: \overline{\mathcal{W}} \rightarrow P_{c p, c v}(\mathcal{X})$ is upper semi-continuous and compact. In view of the choice of $\mathcal{W}$, there is no $x \in \partial \mathcal{W}$ such that $x \in \lambda \Omega(x)$ for some $\lambda \in(0,1)$. Hence, by using Theorem $5, \Omega$ has a fixed point $x \in \overline{\mathcal{W}}$ which is a solution for problem (1). This completes the proof.

Now consider the Banach space $\mathcal{X}=C(I)$ via the norm

$$
\|x\|=\max _{t \in I}|x(t)|+\sum_{i=1}^{m} \max _{t \in I}\left|{ }_{N}^{\mathrm{CF}} \mathcal{D}^{\beta_{i}} x(t)\right|+\sum_{j=1}^{n} \max _{t \in I}\left|{ }^{\mathrm{CF}} \mathcal{I}^{\gamma_{j}} x(t)\right| .
$$

Here, we investigate the fractional integro-differential inclusion

$$
\begin{aligned}
{ }_{N}^{\mathrm{CF}} \mathcal{D}^{\zeta} x(t) \in & \mathcal{F}(t, x(t),(\phi x)(t),(\varphi x)(t), \\
& { }_{N} \mathcal{D}^{\beta_{1}} x(t),{ }_{N}{ }_{N} \mathcal{D}^{\beta_{2}} x(t), \ldots,{ }_{N}{ }^{{ }_{F}} \mathcal{D}^{\beta_{m}} x(t), \\
& \left.{ }^{\mathrm{CF}} \mathcal{I}^{\gamma_{1}} x(t),{ }^{{ }^{C F}} \mathcal{I}^{\gamma_{2}} x(t), \ldots,{ }^{\mathrm{CF}} \mathcal{I}^{\gamma_{n}} x(t)\right),
\end{aligned}
$$

with boundary condition $x(0)=0$, where $\zeta, \beta_{1}, \ldots, \beta_{m}, \gamma_{1}, \ldots, \gamma_{n} \in(0,1)$. Similar to the last case, we say that a function $x \in C(I, \mathbb{R})$ is a solution for problem (2) whenever there exists a function $f \in L^{1}(I)$ such that

$$
\begin{aligned}
f(t) \in & \mathcal{F}\left(t, x(t),(\phi x)(t),(\varphi x)(t),{ }_{N}^{\mathrm{CF}} \mathcal{D}^{\beta_{1}} x(t),{ }_{N}^{\mathrm{CF}} \mathcal{D}^{\beta_{2}} x(t), \ldots,\right. \\
& \left.\left.{ }_{N}^{\mathrm{CF}} \mathcal{D}^{\beta_{m}} x(t)\right),{ }^{\mathrm{CF}} \mathcal{I}^{\gamma_{1}} x(t),{ }^{\mathrm{CF}} \mathcal{I}^{\gamma_{2}} x(t), \ldots,{ }^{\mathrm{CF}} \mathcal{I}^{\gamma_{n}} x(t)\right)
\end{aligned}
$$

for almost all $t \in I$ and $x(t)=a_{\zeta} f(t)+b_{\zeta} \int_{0}^{t} f(s) d s$ for all $t \in I$.

Theorem 12 Assume that $\mathcal{F}: I \times \mathbb{R}^{m+n+3} \rightarrow P_{c v, c p}(\mathbb{R})$ is a multifunction such that the map $t \rightarrow \mathcal{F}\left(t, x_{1}, x_{2}, \ldots, x_{3+m+n}\right)$ is measurable for all $x_{1}, x_{2}, \ldots, x_{m+n+3} \in \mathbb{R}$, the map $t \rightarrow$ $d_{H}(0, \mathcal{F}(t, 0, \ldots, 0))$ is integrably bounded for almost all $t \in I$ and

$$
\begin{aligned}
H_{d}( & \mathcal{F}\left(t, x_{1}, x_{2}, x_{3}, y_{1}, y_{2}, \ldots, y_{m}, z_{1}, z_{2}, \ldots, z_{n}\right), \\
& \left.\mathcal{F}\left(t, x_{1}^{\prime}, x_{2}^{\prime}, x_{3}^{\prime}, y_{1}^{\prime}, y_{2}^{\prime}, \ldots, y_{m}^{\prime}, z_{1}^{\prime}, z_{2}^{\prime}, \ldots, z_{n}^{\prime}\right)\right) \\
\leq & \eta(t)\left(\left|x_{1}-x_{1}^{\prime}\right|+\left|x_{2}-x_{2}^{\prime}\right|+\left|x_{3}-x_{3}^{\prime}\right|+\sum_{i=1}^{m}\left|y_{i}-y_{i}^{\prime}\right|+\sum_{j=1}^{n}\left|z_{j}-z_{j}^{\prime}\right|\right)
\end{aligned}
$$


for all $t \in I$ and all $x_{1}, x_{2}, x_{3}, x_{1}^{\prime}, x_{2}^{\prime}, x_{3}^{\prime}, y_{1}, \ldots, y_{m}, y_{1}^{\prime}, \ldots, y_{m}^{\prime}, z_{1}, \ldots, z_{n}, z_{1}^{\prime}, \ldots, z_{n}^{\prime} \in \mathbb{R}$. If $\Delta \leq 1$, then the inclusion problem (2) has at least one solution, where

$$
\Delta=\eta^{*}\left(1+n+\zeta_{0}+\iota_{0}+\sum_{i=1}^{m} \frac{B\left(\beta_{i}\right)}{\left(1-\beta_{i}\right)^{2}}\right)\left(1+n+\sum_{i=1}^{m} \frac{B\left(\beta_{i}\right)}{\left(1-\beta_{i}\right)^{2}}\right) .
$$

Proof By using the assumptions of Theorem III-6 in [34], we conclude that $\mathcal{F}$ admits a measurable selection $f: I \rightarrow \mathbb{R}$. Since $\mathcal{F}$ is integrable bounded, $f \in L^{1}(I, \mathbb{R})$ and so $S_{\mathcal{F}, x}$ is nonempty for all $x \in \mathcal{X}$, where

$$
\begin{aligned}
S_{\mathcal{F}, x}=\{ & f \in L^{1}(I, R): f(t) \in \mathcal{F}\left(t, x(t),(\phi x)(t),(\varphi x)(t),{ }_{N}{ }^{\mathrm{CF}} \mathcal{D}^{\beta_{1}} x(t),\right. \\
& { }_{N}{ }^{\mathrm{CF}} \mathcal{D}^{\beta_{2}} x(t), \ldots,{ }_{N}{ }^{\mathrm{CF}} \mathcal{D}^{\beta_{m}} x(t),{ }^{\mathrm{CF}} \mathcal{I}^{\gamma_{1}} x(t),{ }^{\mathrm{CF}} \mathcal{I}^{\gamma_{2}} x(t), \ldots, \\
& \left.\left.{ }^{\mathrm{CF}} \mathcal{I}^{\gamma_{n}} x(t)\right) \text { for all } t \in I\right\} .
\end{aligned}
$$

Define the operator $\Omega: \mathcal{X} \rightarrow P(\mathcal{X})$ by

$$
\begin{array}{r}
\Omega(x)=\left\{g \in \mathcal{X}: \text { there exists } f \in S_{\mathcal{F}, x}\right. \text { such that } \\
\left.g(t)=a_{\zeta} f(t)+b_{\zeta} \int_{0}^{t} f(s) d s \text { for all } t \in I\right\} .
\end{array}
$$

First, we show that $\Omega(x) \in P_{c l}(\mathcal{X})$ for all $x \in \mathcal{X}$. Let $g_{n} \in \Omega(x)$ for all $n \geq 0$ and $g_{n} \rightarrow g_{*}$ for some $g \in \mathcal{X}$. For each $\mathrm{n}$, choose $f_{n} \in S_{\mathcal{F}, x}$ such that $g_{n}(t)=a_{\zeta} f_{n}(t)+b_{\zeta} \int_{0}^{t} f_{n}(s) d s$ for all $t \in I$. Since $\mathcal{F}$ has compact values, there is a subsequence of $f_{n}$ that converges to $f$ in $L^{1}(I, \mathbb{R})$. Thus, $f \in S_{\mathcal{F}, x}$ and $g_{n}(t) \rightarrow g_{*}(t)=a_{\zeta} f(t)+b_{\zeta} \int_{0}^{t} f(s) d s$ for all $t \in I$. This implies that $g_{*} \in \Omega$. Now, we show that there exists $\epsilon<1$ such that $H_{d}(\Omega(x), \Omega(y)) \leq \epsilon\|x-y\|$ for all $x, y \in \mathcal{X}$. Let $x, y \in \mathcal{X}$ and $g_{1} \in \Omega(x)$. Choose $f_{1} \in S_{\mathcal{F}, x}$ such that $g_{1}(t)=a_{\zeta} f_{1}(t)+b_{\zeta} \int_{0}^{t} f_{1}(s) d s$ for all $t \in I$. Consider the multifunction $\tilde{\mathcal{F}}$ defined by

$$
\begin{aligned}
\tilde{\mathcal{F}}(t, x(t))= & \mathcal{F}\left(t, x(t),(\phi x)(t),(\varphi x)(t),{ }_{N}{ }^{\mathrm{CF}} \mathcal{D}^{\beta_{1}} x(t),{ }_{N}{ }_{N} \mathcal{D}^{\beta_{2}} x(t), \ldots,{ }_{N}{ }^{\mathrm{CF}} \mathcal{D}^{\beta_{m}} x(t),\right. \\
& \left.{ }^{\mathrm{CF}} \mathcal{I}^{\gamma_{1}} x(t),{ }^{\mathrm{CF}} \mathcal{I}^{\gamma_{2}} x(t), \ldots,{ }^{\mathrm{CF}} \mathcal{I}^{\gamma_{n}} x(t)\right) .
\end{aligned}
$$

Then we have

$$
\begin{aligned}
H_{d}(\tilde{\mathcal{F}}(t, x(t)), \tilde{\mathcal{F}}(t, y(t)) \leq & \eta(t)(|x(t)-y(t)|+|(\phi x)(t)-(\phi y)(t)| \\
& +|(\varphi x)(t)-(\varphi y)(t)| \\
& +\sum_{i=1}^{m}\left|{ }_{N}{ }^{\mathrm{CF}} \mathcal{D}^{\beta_{i}} x(t)-{ }_{N}^{\mathrm{CF}} \mathcal{D}^{\beta_{i}} y(t)\right| \\
& \left.+\sum_{j=1}^{n}\left|{ }^{\mathrm{CF}} \mathcal{I}^{\gamma_{j}} x(t)-{ }^{\mathrm{CF}} \mathcal{I}^{\gamma_{j}} y(t)\right|\right)
\end{aligned}
$$


for almost $t \in I$. Hence, there exists $w_{t} \in \tilde{\mathcal{F}}(t, y(t))$ such that

$$
\begin{aligned}
\left|f_{1}(t)-w_{t}\right| \leq & \eta(t)(|x(t)-y(t)|+|(\phi x)(t)-(\phi y)(t)|+|(\varphi x)(t)-(\varphi y)(t)| \\
& +\sum_{i=1}^{m}\left|{ }_{N}^{\mathrm{CF}} \mathcal{D}^{\beta_{i}} x(t)-{ }_{N}^{\mathrm{CF}} \mathcal{D}^{\beta_{i}} y(t)\right| \\
& \left.+\sum_{j=1}^{n}{ }^{\mathrm{CF}} \mathcal{I}^{\gamma_{j}} x(t)-{ }^{\mathrm{CF}} \mathcal{I}^{\gamma_{j}} y(t) \mid\right):=M_{t}
\end{aligned}
$$

for almost $t \in I$. Define $V: I \rightarrow P(\mathbb{R})$ by $V(t)=\left\{u \in \mathbb{R}:\left|f_{1}(t)-u\right| \leq M_{t}\right\}$ for all $t \in I$. By using Theorem III-41 in [34], we get $V$ is measurable. Since $t \mapsto V(t) \cap \tilde{\mathcal{F}}(t, y(t))$ is measurable (Proposition III-4 in [34]), we can choose $f_{2} \in S_{\mathcal{F}, y}$ such that $\left|f_{1}(t)-f_{2}(t)\right| \leq M_{t}$ for almost all $t \in I$. Define $g_{2} \in \Omega(y)$ by $g_{2}(t)=a_{\zeta} f_{2}(t)+b_{\zeta} \int_{0}^{t} f_{2}(s) d s$ for all $t \in I$. Then we have

$$
\begin{aligned}
\left\|g_{1}-g_{2}\right\|= & \max _{t \in I}\left|g_{1}(t)-g_{2}(t)\right|+\sum_{i=1}^{m} \max _{t \in I}\left|{ }_{N}^{\mathrm{CF}} \mathcal{D}^{\beta_{i}} g_{1}(t)-{ }_{N}{ }_{N} \mathcal{D}^{\beta_{i}} g_{2}(t)\right| \\
& +\sum_{i=1}^{n} \max _{t \in I}\left|{ }^{\mathrm{CF}} \mathcal{I}^{\gamma_{i}} g_{1}(t)-{ }^{\mathrm{CF}} \mathcal{I}^{\gamma_{i}} g_{2}(t)\right|\left|g_{1}(t)-g_{2}(t)\right| \\
\leq & a_{\zeta}\left|f_{1}(t)-f_{2}(t)\right|+b_{\zeta} \int_{0}^{t}\left|f_{1}(s)-f_{2}(s)\right| d s \\
\leq & \eta(t)\left(1+n+\zeta_{0}+\iota_{0}+\sum_{i=1}^{m} \frac{B\left(\beta_{i}\right)}{\left(1-\beta_{i}\right)^{2}}\right)\left(a_{\zeta}+b_{\zeta}\right)\|x-y\|,
\end{aligned}
$$

and so

$$
\begin{aligned}
& \left|{ }_{N}^{\mathrm{CF}} \mathcal{D}^{\beta_{i}} g_{1}(t)-{ }_{N}^{\mathrm{CF}} \mathcal{D}^{\beta_{i}} g_{2}(t)\right| \\
& \quad \leq \frac{B\left(\beta_{i}\right)}{\left(1-\beta_{i}\right)^{2}}\left|g_{1}(t)-g_{2}(t)\right| \\
& \quad \leq \eta(t) \frac{B\left(\beta_{i}\right)}{\left(1-\beta_{i}\right)^{2}}\left(1+n+\zeta_{0}+\iota_{0}+\sum_{i=1}^{m} \frac{B\left(\beta_{i}\right)}{\left(1-\beta_{i}\right)^{2}}\right)\left(a_{\zeta}+b_{\zeta}\right)\|x-y\| .
\end{aligned}
$$

Thus,

$$
\begin{aligned}
\left|{ }^{\mathrm{CF}} \mathcal{I}^{\gamma_{i}} g_{1}(t)-{ }^{\mathrm{CF}} \mathcal{I}^{\gamma_{i}} g_{2}(t)\right| & \leq\left|g_{1}(t)-g_{2}(t)\right| \\
& \leq \eta(t)\left(1+n+\zeta_{0}+\iota_{0}+\sum_{i=1}^{m} \frac{B\left(\beta_{i}\right)}{\left(1-\beta_{i}\right)^{2}}\right)\left(a_{\zeta}+b_{\zeta}\right)\|x-y\|,
\end{aligned}
$$

and so

$$
\begin{aligned}
\left\|g_{1}-g_{2}\right\| \leq & \eta^{*}\left(1+n+\zeta_{0}+\iota_{0}+\sum_{i=1}^{m} \frac{B\left(\beta_{i}\right)}{\left(1-\beta_{i}\right)^{2}}\right) \\
& \times\left(1+n+\sum_{i=1}^{m} \frac{B\left(\beta_{i}\right)}{\left(1-\beta_{i}\right)^{2}}\right)\|x-y\|=\Delta\|x-y\| .
\end{aligned}
$$


Hence, $H_{d}(\Omega(x), \Omega(y)) \leq \Delta\|x-y\|$. Since $\Delta<1, \Omega$ is a closed-valued contraction. By using Theorem $6, \Omega$ has a fixed point which is a solution for the inclusion problem (2).

Consider the Banach space $\mathcal{X}=\left\{x: x,{ }_{N}^{C F} \mathcal{D}^{\beta_{i}} x \in C(I, \mathbb{R})\right\}$ endowed with the norm $\|x\|=$ $\max _{t \in I}|x(t)|+\left.\max _{t \in I}\right|_{N} ^{C F} \mathcal{D}^{\beta_{i}} x(t) \mid$. Here, we review the inclusion problem

$$
\begin{aligned}
{ }_{N}^{\mathrm{CF}} \mathcal{D}^{\zeta} x(t) \in & \mathcal{F}\left(t, x(t),(\phi x)(t),{ }_{N}^{\mathrm{CF}} \mathcal{D}^{\beta_{1}} x(t),{ }_{N}{ }_{N} \mathcal{D}^{\beta_{2}} x(t), \ldots,{ }_{N}{ }^{\mathrm{CF}} \mathcal{D}^{\beta_{n}} x(t)\right) \\
& +\mathcal{G}\left(t, x(t),(\varphi x)(t),{ }^{\mathrm{CF}} \mathcal{I}^{\beta_{1}} x(t),{ }^{\mathrm{CF}} \mathcal{I}^{\beta_{2}} x(t), \ldots,{ }^{\mathrm{CF}} \mathcal{I}^{\beta_{n}} x(t)\right)
\end{aligned}
$$

with boundary condition $x(0)=0$, where $\zeta, \beta_{1}, \ldots, \beta_{n} \in(0,1)$. Define the set of the selections of $\mathcal{F}$ and $\mathcal{G}$ at $x$ by

$$
\begin{aligned}
S_{\mathcal{F}, x}= & \left\{v \in L^{1}[0,1]: v(t) \in \mathcal{F}(t, x(t),(\phi x)(t),\right. \\
& \left.\left.{ }_{N}^{\mathrm{CF}} \mathcal{D}^{\beta_{1}} x(t),{ }_{N}^{\mathrm{CF}} \mathcal{D}^{\beta_{2}} x(t), \ldots,{ }_{N}^{\mathrm{CF}} \mathcal{D}^{\beta_{n}} x(t)\right) \text { for almost all } t \in I\right\}
\end{aligned}
$$

and

$$
\begin{aligned}
S_{\mathcal{G}, x}= & \left\{v \in L^{1}[0,1]: v(t) \in \mathcal{G}(t, x(t),(\varphi x)(t),\right. \\
& \left.\left.{ }^{\mathrm{CF}} \mathcal{I}^{\beta_{1}} x(t),{ }^{\mathrm{CF}} \mathcal{I}^{\beta_{2}} x(t), \ldots,{ }^{\mathrm{CF}} \mathcal{I}^{\beta_{n}} x(t)\right) \text { for almost all } t \in I\right\} .
\end{aligned}
$$

We suppose that $S_{\mathcal{F}, x} \neq \emptyset$ and $S_{\mathcal{G}, x} \neq \emptyset$ for all $x \in \mathcal{X}$. A function $x \in C(I, \mathbb{R})$ is a solution for problem (3) whenever there exist two functions $f \in H^{1}(I)$ and $f^{\prime} \in H^{1}(I)$ such that

$$
f(t) \in \mathcal{F}\left(t, x(t),(\phi x)(t),{ }_{N}^{\mathrm{CF}} \mathcal{D}^{\beta_{1}} x(t),{ }_{N}^{\mathrm{CF}} \mathcal{D}^{\beta_{2}} x(t), \ldots,{ }_{N}^{\mathrm{CF}} \mathcal{D}^{\beta_{n}} x(t)\right)
$$

and $f^{\prime} \in \mathcal{G}\left(t, x(t),(\varphi x)(t),{ }^{\mathrm{CF}} \mathcal{I}^{\beta_{1}} x(t),{ }^{\mathrm{CF}} \mathcal{I}^{\beta_{2}} x(t), \ldots,{ }^{\mathrm{CF}} \mathcal{I}^{\beta_{n}} x(t)\right)$ for almost all $t \in I$ and

$$
x(t)=a_{\zeta} f(t)+b_{\zeta} \int_{0}^{t} f(s) d s+a_{\zeta} f^{\prime}(t)+b_{\zeta} \int_{0}^{t} f^{\prime}(s) d s
$$

for all $t \in I$.

Theorem 13 Let $\mathcal{F}: I \times \mathbb{R}^{n+2} \rightarrow P_{c p, c v}(\mathbb{R})$ be a multifunction and $\mathcal{G}: I \times \mathbb{R}^{n+2} \rightarrow P_{c p, c v}(\mathbb{R})$ be a Caratheodory set-valued map. Assume that there exist continuous functions $p, m: I \rightarrow$ $(0, \infty)$ and $\eta(t) \in L^{\infty}(I)$ such that $t \vdash \mathcal{F}\left(t, y_{1}, \ldots, y_{n+2}\right)$ is measurable,

$$
\begin{gathered}
\left\|\mathcal{F}\left(t, x(t),(\phi x)(t),{ }_{N}{ }^{\mathrm{CF}} \mathcal{D}^{\beta_{1}} x(t),{ }_{N}{ }_{N} \mathcal{D}^{\beta_{2}} x(t), \ldots,{ }_{N}{ }^{\mathrm{CF}} \mathcal{D}^{\beta_{n}} x(t)\right)\right\| \leq m(t), \\
\left\|\mathcal{G}\left(t, x(t),(\varphi x)(t),{ }^{\mathrm{CF}} \mathcal{I}^{\beta_{1}} x(t),{ }^{\mathrm{CF}} \mathcal{I}^{\beta_{2}} x(t), \ldots,{ }^{\mathrm{CF}} \mathcal{I}^{\beta_{n}} x(t)\right)\right\| \leq p(t),
\end{gathered}
$$

and

$$
H_{d}\left(\mathcal{F}\left(t, y_{1}, \ldots, y_{n+2}\right), \mathcal{F}\left(t, y_{1}^{\prime}, \ldots, y_{n+2}^{\prime}\right)\right) \leq \eta(t) \sum_{i=1}^{n+2}\left(\left|y_{i}-y_{i}^{\prime}\right|\right)
$$

for all $t \in I, x \in \mathcal{X}$ and $y_{1}, \ldots, y_{n+2}, y_{1}^{\prime}, \ldots, y_{n+2}^{\prime} \in \mathbb{R}$. If $L=\eta^{*}\left(1+\sum_{i=1}^{n} \frac{B\left(\beta_{i}\right)}{\left(1-\beta_{i}\right)^{2}}\right)\left(1+\zeta_{0}+\right.$ $\left.\sum_{i=1}^{n} \frac{B\left(\beta_{i}\right)}{\left(1-\beta_{i}\right)^{2}}\right)<1$, then the inclusion problem (3) has at least one solution. 
Proof Put $\mathcal{Y}=\{x \in \mathcal{X}:\|x\| \leq M\}$, where $M=\left(1+\sum_{i=1}^{n} \frac{B\left(\beta_{i}\right)}{\left(1-\beta_{i}\right)^{2}}\right)\left(\|p\|_{\infty}+\|m\|_{\infty}\right)$. One can check that $\mathcal{Y}$ is a closed, bounded, and convex subset of $\mathcal{X}$. Define the multivalued operators $\mathcal{A}, \mathcal{B}: \mathcal{Y} \rightarrow P(\mathcal{X})$ by

$$
\mathcal{A} x:=\left\{x \in \mathcal{X}: \text { there is } v \in S_{\mathcal{F}, x} \text { such that } x(t)=a_{\zeta} v(t)+b_{\zeta} \int_{0}^{t} v(s) d s \text { for all } t \in I\right\}
$$

and $\mathcal{B} x:=\left\{x \in \mathcal{X}:\right.$ there is $v \in S_{\mathcal{G}, x}$ such that $x(t)=a_{\zeta} v(t)+b_{\zeta} \int_{0}^{t} v(s) d s$ for all $\left.t \in I\right\}$. Note that problem (3) is equivalent to the inclusion fixed point problem $x \in \mathcal{A} x+\mathcal{B} x$. Also, the operator $\mathcal{A}$ is equivalent to the composition $\theta \circ S_{\mathcal{F}}$, where $\theta$ is the continuous linear operator on $L^{1}(0,1)$ into $\mathcal{X}$ defined by $\theta v(t)=a_{\zeta} v(t)+b_{\zeta} \int_{0}^{t} v(s) d s$. Let $x \in \mathcal{Y}$ and $\left\{v_{n}\right\}_{n \geq 1}$ be a sequence in $S_{\mathcal{F}, x}$. Then $v_{n}(t) \in \mathcal{F}\left(t, x(t),(\phi x)(t),{ }^{\mathrm{CF}} \mathcal{D}^{\beta_{1}} x(t),{ }^{\mathrm{CF}} \mathcal{D}^{\beta_{2}} x(t), \ldots,{ }^{\mathrm{CF}} \mathcal{D}^{\beta_{n}} x(t)\right)$ for almost $t \in I$. Since

$$
\mathcal{F}\left(t, x(t),(\phi x)(t),{ }_{N}^{\mathrm{CF}} \mathcal{D}^{\beta_{1}} x(t),{ }_{N}^{\mathrm{CF}} \mathcal{D}^{\beta_{2}} x(t), \ldots,{ }_{N}^{\mathrm{CF}} \mathcal{D}^{\beta_{n}} x(t)\right)
$$

is compact for all $t \in I$, there is a convergent subsequence of $\left\{v_{n}(t)\right\}$ (call it again $\left\{v_{n}(t)\right\}$ ) such that it converges in measure to some $v(t) \in S_{\mathcal{F}, x}$ for almost all $t \in I$. Since $\theta$ is continuous, $\theta v_{n}(t) \rightarrow \theta v(t)$ pointwise on $I$. In order to show that the convergence is uniform, we show that $\left\{\theta v_{n}\right\}$ is an equi-continuous sequence. Let $\tau<t \in I$. Then we have

$$
\left|\theta v_{n}(t)-\theta v_{n}(\tau)\right| \leq a_{\zeta}\left|v_{n}(t)-v_{n}(\tau)\right|+b_{\zeta} \int_{\tau}^{t}\left|v_{n}(s)\right| d s
$$

Since the right-hand of the above inequality tends to 0 as $t \rightarrow \tau$, the sequence $\left\{\theta v_{n}\right\}$ is equicontinuous. Now, by using the Arzela-Ascoli theorem, there is a uniformly convergent subsequence of $\left\{v_{n}\right\}$ (we show it again by $\left\{v_{n}\right\}$ ) such that $\theta v_{n} \rightarrow \theta v$. Note that $\theta v \in \theta\left(S_{\mathcal{F}, x}\right)$. Hence, $\mathcal{A} x=\theta\left(S_{\mathcal{F}, x}\right)$ is compact for all $x \in \mathcal{Y}$. Now, we show that $\mathcal{A} x$ is convex for all $x \in \mathcal{Y}$. Let $u, u^{\prime} \in \mathcal{A} x$. Choose $v, v^{\prime} \in S_{\mathcal{F}, x}$ such that $u(t)=a_{\zeta} v(t)+b_{\zeta} \int_{0}^{t} v(s) d s$ and $u^{\prime}(t)=$ $a_{\zeta} v^{\prime}(t)+b_{\zeta} \int_{0}^{t} v^{\prime}(s) d s$ for almost all $t \in I$. Let $0 \leq \lambda \leq 1$. Then we have

$$
\left(\lambda u+(1-\lambda) u^{\prime}\right)(t)=a_{\zeta}\left(\lambda v(t)+(1-\lambda) v^{\prime}(t)\right)+b_{\zeta} \int_{0}^{t}\left(\lambda v(s)+(1-\lambda) v^{\prime}(s)\right) d s .
$$

Since $\mathcal{F}$ is convex-valued, $\lambda u+(1-\lambda) u^{\prime} \in \mathcal{A} x$. Similarly, we can show that $\mathcal{B}$ is compact and convex-valued. Here, we show that $\mathcal{A} y+\mathcal{B} y \subset \mathcal{Y}$ for all $y \in \mathcal{Y}$. Let $y \in \mathcal{Y}, u \in \mathcal{A} y$, and $u^{\prime} \in \mathcal{B} y$. Choose $v \in S_{\mathcal{F}, y}$ and $v^{\prime} \in S_{\mathcal{G}, y}$ such that $u(t)=a_{\zeta} v(t)+b_{\zeta} \int_{0}^{t} v(s) d s$ and $u^{\prime}(t)=$ $a_{\zeta} v^{\prime}(t)+b_{\zeta} \int_{0}^{t} v^{\prime}(s) d s$ for almost all $t \in I$. Hence,

$$
\left|u(t)+u^{\prime}(t)\right| \leq a_{\zeta}\left(|v(t)|+\left|v^{\prime}(t)\right|\right)+b_{\zeta} \int_{0}^{t}\left(|v(s)|+\left|v^{\prime}(s)\right|\right) d s
$$

and so

$$
\begin{aligned}
\left|{ }_{N}^{\mathrm{CF}} \mathcal{D}^{\beta_{i}} u(t)+{ }_{N}^{\mathrm{CF}} \mathcal{D}^{\beta_{i}} u^{\prime}(t)\right| \leq & \left|{ }_{N}^{\mathrm{CF}} \mathcal{D}^{\beta_{i}} u(t)\right|+\left|{ }_{N}^{\mathrm{CF}} \mathcal{D}^{\beta_{i}} u^{\prime}(t)\right| \\
\leq & \frac{a_{\zeta} B\left(\beta_{i}\right)}{\left(1-\beta_{i}\right)^{2}}(p(t)+m(t)) \\
& +\frac{b_{\zeta} B\left(\beta_{i}\right)}{\left(1-\beta_{i}\right)^{2}}\left(\|p\|_{\infty}+\|m\|_{\infty}\right)
\end{aligned}
$$


for $1 \leq i \leq n$. This implies that

$$
\max _{t \in I}\left|u(t)+u^{\prime}(t)\right| \leq a_{\zeta}\left(\|p\|_{\infty}+\|m\|_{\infty}\right)+b_{\zeta}\left(\|p\|_{\infty}+\|m\|_{\infty}\right)=\|p\|_{\infty}+\|m\|_{\infty}
$$

and

$$
\begin{aligned}
\max _{t \in I}\left|{ }_{N}^{\mathrm{CF}} \mathcal{D}^{\beta_{i}} u(t)+{ }_{N}^{\mathrm{CF}} \mathcal{D}^{\beta_{i}} u^{\prime}(t)\right| \leq & \frac{a_{\zeta} B\left(\beta_{i}\right)}{\left(1-\beta_{i}\right)^{2}}\left(\|p\|_{\infty}+\|m\|_{\infty}\right) \\
& +\frac{b_{\zeta} B\left(\beta_{i}\right)}{\left(1-\beta_{i}\right)^{2}}\left(\|p\|_{\infty}+\|m\|_{\infty}\right) \\
= & \frac{B\left(\beta_{i}\right)\left(\|p\|_{\infty}+\|m\|_{\infty}\right)}{\left(1-\beta_{i}\right)^{2}} .
\end{aligned}
$$

Thus, $\left\|u+u^{\prime}\right\| \leq\left(1+\sum_{i=1}^{n}\left(\frac{B\left(\beta_{i}\right)}{\left(1-\beta_{i}\right)^{2}}\right)\right)\left(\|p\|_{\infty}+\|m\|_{\infty}\right)=M$. Now, we show that the operator $\mathcal{B}$ is compact on $\mathcal{Y}$. To do this, we prove that $\mathcal{B}(\mathcal{Y})$ is uniformly bounded and equicontinuous in $\mathcal{X}$. Let $u \in \mathcal{B}(\mathcal{Y})$ be arbitrary. Choose $v \in S_{\mathcal{G}, x}$ such that $u(t)=a_{\zeta} v(t)+$ $b_{\zeta} \int_{0}^{t} v(s) d s$ for some $x \in \mathcal{Y}$. Hence,

$$
\begin{aligned}
|u(t)| & \left.\leq a_{\zeta}|v(t)|+\left.b_{\zeta} \int_{0}^{t}|v(s)| d s\right|_{N} ^{\mathrm{CF}} \mathcal{D}^{\beta_{i}} u(t)\right) \mid \\
& \leq\left. a_{\zeta}\right|_{N} ^{\mathrm{CF}} \mathcal{D}^{\beta_{i}} v(t)\left|+b_{\zeta} \int_{0}^{t}\right|{ }_{N}^{\mathrm{CF}} \mathcal{D}^{\beta_{i}} v(s) \mid d s \\
& \leq \frac{B\left(\beta_{i}\right)\left(a_{\zeta}+b_{\zeta}\right)}{\left(1-\beta_{i}\right)^{2}} p(t) \\
& =\frac{B\left(\beta_{i}\right)}{\left(1-\beta_{i}\right)^{2}} p(t) .
\end{aligned}
$$

Thus, $\max _{t \in I}|u(t)| \leq\left(a_{\zeta}+b_{\zeta}\right)\|p\|_{\infty}=\|p\|_{\infty}$ and $\left.\max _{t \in I}\right|_{N} ^{\mathrm{CF}} \mathcal{D}^{\beta_{i}} u_{i}(t) \mid \leq \frac{B\left(\beta_{i}\right)}{\left(1-\beta_{i}\right)^{2}}\|p\|_{\infty}$ for $i=1, \ldots, n$, and so $\|u\| \leq\left(1+\sum_{i=1}^{n} \frac{B\left(\beta_{i}\right)}{\left(1-\beta_{i}\right)^{2}}\right)\|p\|_{\infty}$. Here, we show that $\mathcal{B}$ maps $\mathcal{Y}$ to equicontinuous subsets of $\mathcal{X}$. Let $t, \tau \in I$ with $\tau<t, x \in \mathcal{Y}$ and $u \in \mathcal{B} x$. Choose $v \in S_{\mathcal{G}, x}$ such that $u(t)=a_{\zeta} v(t)+b_{\zeta} \int_{0}^{t} v(s) d s$ for all. Then we have

$$
|u(t)-u(\tau)| \leq a_{\zeta}(v(t)-v(\tau))+b_{\zeta} \int_{\tau}^{t} v(s) d s \leq a_{\zeta}(v(t)-v(\tau))+b_{\zeta}(t-\tau)\|p\|_{\infty}
$$

and $\left.\right|_{N} ^{\mathrm{CF}} \mathcal{D}^{\beta_{i}} u(t)-{ }_{N}^{\mathrm{CF}} \mathcal{D}^{\beta_{i}} u(\tau)\left|\leq \frac{B\left(\beta_{i}\right)}{\left(1-\beta_{i}\right)^{2}}\right| u(t)-u(\tau) \mid$. Since the right-hand of the inequality tends to 0 as $t \rightarrow \tau$, by using the Arzela-Ascoli theorem, we get $\mathcal{B}$ is compact. Now, we show that $\mathcal{B}$ has a closed graph. Let $x_{n} \in \mathcal{Y}$ and $u_{n} \in \mathcal{B}\left(x_{n}\right)$ for all $n$ with $x_{n} \rightarrow x_{0}$ and $u_{n} \rightarrow u_{0}$. We show that $u_{0} \in \mathcal{B}\left(x_{0}\right)$. For each $n$, choose $v_{n} \in S_{\mathcal{G}, x_{n}}$ such that $u_{n}(t)=a_{\zeta} v_{n}(t)+$ $b_{\zeta} \int_{0}^{t} v_{n}(s) d s$ for all $t \in I$. Again, consider the continuous linear operator $\theta: L^{1}(0,1) \rightarrow$ $\mathcal{X}$ defined by $\theta(v)(t)=a_{\zeta} v(t)+b_{\zeta} \int_{0}^{t} v(s) d s$. By using Lemma $4, \theta o S_{\mathcal{G}}$ is a closed graph operator. Since $u_{n} \in \theta\left(S_{\mathcal{G}, x_{n}}\right)$ for all $n$ and $x_{n} \rightarrow x_{0}$, there exists $v_{0} \in S_{\mathcal{G}, x_{0}}$ such that $u_{0}(t)=$ $a_{\zeta} v_{0}(t)+b_{\zeta} \int_{0}^{t} v_{0}(s) d s$. Hence, $u_{0} \in \mathcal{B}\left(x_{0}\right)$. This implies that $\mathcal{B}$ has a closed graph, and so $\mathcal{B}$ is upper semi-continuous. Now, we show that $\mathcal{A}$ is a contraction multifunction. Let $x, y \in \mathcal{X}$ 
and $u \in \mathcal{A} y$. Choose $v \in S_{\mathcal{F}, y}$ such that $u(t)=a_{\zeta} v(t)+b_{\zeta} \int_{0}^{t} v(s) d s$ for all $t \in I$. Since

$$
\begin{aligned}
& H_{d}\left(\mathcal{F}\left(t, x(t),(\phi x)(t),{ }_{N}{ }_{N} \mathcal{D}^{\beta_{1}} x(t), \ldots,{ }_{N}{ }_{N} \mathcal{D}^{\beta_{n}} x(t)\right),\right. \\
& \left.\quad \mathcal{F}\left(t, y(t),(\phi y)(t),{ }_{N} \mathcal{C F}^{\beta_{1}} y(t), \ldots,{ }_{N}{ }^{\mathrm{CF}} \mathcal{D}^{\beta_{n}} y(t)\right)\right) \\
& \leq \eta(t)\left(1+\zeta_{0}+\sum_{i=1}^{n} \frac{B\left(\beta_{i}\right)}{\left(1-\beta_{i}\right)^{2}}\right)\|x-y\|
\end{aligned}
$$

for almost all $t \in I$, there exists $w \in \mathcal{F}\left(t, x(t),(\phi x)(t),{ }_{N}^{\mathrm{CF}} \mathcal{D}^{\beta_{1}} x(t), \ldots,{ }_{N}^{\mathrm{CF}} \mathcal{D}^{\beta_{n}} x(t)\right)$ such that $|v(t)-w| \leq \eta(t)\left(1+\zeta_{0}+\sum_{i=1}^{n} \frac{B\left(\beta_{i}\right)}{\left(1-\beta_{i}\right)^{2}}\right)\|x-y\|$ for almost all $t \in I$. Consider the multifunction $U: I \rightarrow 2^{\mathbb{R}}$ defined by

$$
U(t)=\left\{w \in \mathbb{R}:|v(t)-w| \leq \eta(t)\left(1+\zeta_{0}+\sum_{i=1}^{n} \frac{B\left(\beta_{i}\right)}{\left(1-\beta_{i}\right)^{2}}\right)\|x-y\| \text { for almost all } t \in I\right\} .
$$

Since $v$ and $\eta\left(1+\zeta_{0}+\sum_{i=1}^{n} \frac{B\left(\beta_{i}\right)}{\left(1-\beta_{i}\right)^{2}}\right)$ are measurable, we get

$$
U(\cdot) \cap \mathcal{F}\left(t, x(\cdot),(\phi x)(\cdot),{ }^{\mathrm{CF}} \mathcal{D}^{\beta_{1}} x(\cdot), \ldots,{ }^{\mathrm{CF}} \mathcal{D}^{\beta_{n}} x(\cdot)\right)
$$

is a measurable multifunction. Choose

$$
v^{\prime}(t) \in \mathcal{F}\left(t, x(t),(\phi x)(t),{ }_{N}{ }_{N} \mathcal{D}^{\beta_{1}} x(t), \ldots,{ }_{N}{ }^{\mathrm{CF}} \mathcal{D}^{\beta_{n}} x(t)\right)
$$

such that $\left|v(t)-v^{\prime}(t)\right| \leq \eta(t)\left(1+\zeta_{0}+\sum_{i=1}^{n} \frac{B\left(\beta_{i}\right)}{\left(1-\beta_{i}\right)^{2}}\right)\|x-y\|$ and $u^{\prime}(t)=a_{\zeta} v^{\prime}(t)+b_{\zeta} \int_{0}^{t} v^{\prime}(s) d s$ for all $t \in I$. Since $\left|u(t)-u^{\prime}(t)\right| \leq a_{\zeta}\left(v(t)-v^{\prime}(t)\right)+b_{\zeta} \int_{0}^{t}\left(v(s)-v^{\prime}(s)\right) d s$ and

$$
\left|{ }_{N}^{\mathrm{CF}} \mathcal{D}^{\beta_{i}} u(t)-{ }_{N}^{\mathrm{CF}} \mathcal{D}^{\beta_{i}} u^{\prime}(t)\right| \leq \frac{B\left(\beta_{i}\right)}{\left(1-\beta_{i}\right)^{2}}\left|u(t)-u^{\prime}(t)\right|
$$

we get

$$
\begin{aligned}
\max _{t \in I}\left|u(t)-u^{\prime}(t)\right| \leq & a_{\zeta} \eta^{*}\left(1+\zeta_{0}+\sum_{i=1}^{n} \frac{B\left(\beta_{i}\right)}{\left(1-\beta_{i}\right)^{2}}\right)\|x-y\| \\
& +b_{\zeta} \eta^{*}\left(1+\zeta_{0}+\sum_{i=1}^{n} \frac{B\left(\beta_{i}\right)}{\left(1-\beta_{i}\right)^{2}}\right)\|x-y\| \\
= & \eta^{*}\left(1+\zeta_{0}+\sum_{i=1}^{n} \frac{B\left(\beta_{i}\right)}{\left(1-\beta_{i}\right)^{2}}\right)\|x-y\|
\end{aligned}
$$

and

$$
\max _{t \in I}\left|{ }_{N}^{\mathrm{CF}} \mathcal{D}^{\beta_{i}} u(t)-{ }_{N}^{\mathrm{CF}} \mathcal{D}^{\beta_{i}} u^{\prime}(t)\right| \leq \eta^{*} \frac{B\left(\beta_{i}\right)}{\left(1-\beta_{i}\right)^{2}}\left(1+\zeta_{0}+\sum_{i=1}^{n} \frac{1}{\left(1-\beta_{i}\right)^{2}}\right)\|x-y\| \|
$$

for $1 \leq i \leq n$. Hence, $\left\|u-u^{\prime}\right\| \leq \eta^{*}\left(1+\sum_{i=1}^{n} \frac{B\left(\beta_{i}\right)}{\left(1-\beta_{i}\right)^{2}}\right)\left(1+\zeta_{0}+\sum_{i=1}^{n} \frac{B\left(\beta_{i}\right)}{\left(1-\beta_{i}\right)^{2}}\right)\|x-y\|$. This implies that $H_{d}(\mathcal{A} x, \mathcal{A} y) \leq L\|x-y\|$. Now, by using Theorem 7 , the inclusion fixed point problem $x \in \mathcal{A} x+\mathcal{B} x$ has a solution which is a solution for the inclusion problem (3). 
Now, we are ready to investigate the fractional integro-differential inclusion

$$
\begin{gathered}
{ }_{N}^{{ }_{N}} \mathcal{D}^{\zeta}\left(\frac{x(t)}{g\left(t, x(t),(\phi x)(t),(\varphi x)(t),{ }_{N}^{\mathrm{CF}} \mathcal{D}{ }^{\zeta_{1}} x(t), \ldots,{ }_{N}^{\mathrm{CF}} \mathcal{D}{ }^{\xi_{n}} x(t)\right)}\right) \\
\quad \in \mathcal{G}\left(t, x(t),(\phi x)(t),(\varphi x)(t),{ }_{N}^{\mathrm{CF}} \mathcal{D}^{\beta_{1}} x(t), \ldots,{ }_{N}^{\mathrm{CF}_{1}} \mathcal{D}^{\beta_{k}} x(t)\right)
\end{gathered}
$$

with boundary condition $u(0)=0$, where $\zeta, \zeta_{1}, \ldots, \zeta_{n}, \beta_{1}, \ldots, \beta_{k} \in(0,1), g: I \times \mathbb{R}^{n+3} \rightarrow$ $\mathbb{R} \backslash\{0\}$ is continuous and $\mathcal{G}: I \times \mathbb{R}^{k+3} \rightarrow \mathcal{P}(\mathbb{R})$ is a multifunction. We say that $x \in \mathcal{X}$ is a solution for problem (4) whenever it satisfies the boundary conditions and there exists $v \in S_{\mathcal{G}, x}$ such that

$$
\begin{aligned}
x(t)= & g\left(t, x(t),(\phi x)(t),(\varphi x)(t),{ }_{N}{ }_{N} \mathcal{D}^{\zeta 1} x(t), \ldots,{ }_{N}{ }^{C F} \mathcal{D}^{\zeta n} x(t)\right) \\
& \times\left(a_{\zeta} v(t)+b_{\zeta} \int_{0}^{t} v(s) d s\right),
\end{aligned}
$$

where

$$
\begin{aligned}
S_{\mathcal{G}, x}= & \left\{v \in L^{1}[0,1]: v(t) \in \mathcal{G}(t, x(t),(\phi x)(t),(\varphi x)(t),\right. \\
& \left.\left.{ }_{N} \mathcal{D}^{{ }^{\beta}} x(t), \ldots,{ }_{N}{ }^{\mathrm{CF}} \mathcal{D}^{\beta_{k}} x(t)\right) \text { for almost all } t \in I\right\} .
\end{aligned}
$$

Theorem 14 Suppose that $\mathcal{G}: I \times \mathbb{R}^{k+3} \rightarrow \mathcal{P}_{c p, c v}(\mathbb{R})$ is a Caratheodory set-valued map, $g: J \times \mathbb{R}^{n+3} \rightarrow \mathbb{R} \backslash\{0\}$ is a bounded continuous map with upper bound $K$ and there are continuous functions $p, m: J \rightarrow(0, \infty)$ such that $\left\|\mathcal{G}\left(t, x_{1}, x_{2}, \ldots, x_{k+3}\right)\right\| \leq m(s)$ and

$$
\left|g\left(t, x_{1}, x_{2}, \ldots, x_{n+3}\right)-g\left(t, y_{1}, y_{2}, \ldots, y_{n+3}\right)\right| \leq \eta(t) \sum_{i=1}^{n+3}\left|x_{i}-y_{i}\right|
$$

for all $t \in I$. If $\eta^{*}\left(1+\zeta_{0}+\iota_{0}+\sum_{i=1}^{n} \frac{B\left(\beta_{i}\right)}{\left(1-\beta_{i}\right)^{2}}\right) \cdot K \cdot\|m\|_{\infty}<1$, then the inclusion problem (4) has a solution.

Proof Put $S=\{x \in \mathcal{X}:\|x\| \leq L\}$, where $L=K\|m\|_{\infty}$. It is clear that $S$ is a convex, closed, and bounded subset of the Banach space $\mathcal{X}$. Define $\mathcal{A}, \mathcal{B}: S \rightarrow \mathcal{P}(\mathcal{X})$ by

$$
\mathcal{A} x(t)=g\left\{t, x(t),(\phi x)(t),(\varphi x)(t),{ }_{N}^{\mathrm{CF}} \mathcal{D}^{\zeta_{1}} x(t), \ldots,{ }_{N}^{\mathrm{CF}} \mathcal{D}^{\zeta_{n}} x(t)\right\}
$$

and

$$
\mathcal{B} x(t)=\left\{u \in \mathcal{X}: \text { there is } v \in S_{\mathcal{G}, x} \text { such that } u(t)=a_{\zeta} v(t)+b_{\zeta} \int_{0}^{t} v(s) d s \text { for all } t \in I\right\} .
$$

Thus, the problem of fractional differential inclusions is equivalent to the inclusion problem $x \in \mathcal{A}(x) \mathcal{B}(x)$. Consider the operator $\mathcal{B}=\theta \circ S_{\mathcal{G}}$, where $\theta$ is the continuous linear operator on $L^{1}(I)$ into $\mathcal{X}$ defined by $\theta v(s)=a_{\zeta} v(t)+b_{\zeta} \int_{0}^{t} v(s) d s$. Let $x \in S$ be arbitrary and $\left\{v_{n}\right\}$ be a sequence in $S_{\mathcal{G}, x}$. Then $v_{n}(t) \in \mathcal{G}\left(t, x(t),(\phi x)(t),(\varphi x)(t),{ }_{N}{ }^{\mathrm{CF}} \mathcal{D}^{\beta_{1}} x(t), \ldots,{ }_{N}{ }_{\mathrm{CF}} \mathcal{D}^{\beta_{k}} x(t)\right)$ for almost $t \in I$. Since

$$
\mathcal{G}\left(t, x(t),(\phi x)(t),(\varphi x)(t),{ }_{N}^{\mathrm{CF}} \mathcal{D}^{\beta_{1}} x(t), \ldots,{ }_{N}^{\mathrm{CF}} \mathcal{D}^{\beta_{k}} x(t)\right)
$$


is compact for all $t \in I$, there is a convergent subsequence of $\left\{v_{n}(t)\right\}$ (show it by $\left\{v_{n}(t)\right\}$ again) to some $v \in S_{\mathcal{G}_{,}, x}$. Note that $\theta v_{n}(t) \rightarrow \theta v(t)$ pointwise on $I$ because $\theta$ is continuous. Now, we show that $\left\{\theta v_{n}\right\}$ is an equi-continuous sequence. Let $\tau<t \in I$. Then we have $\left|\theta v_{n}(t)-\theta v_{n}(\tau)\right| \leq a_{\zeta}\left|v_{n}(t)-v_{n}(\tau)\right|+b_{\zeta} \int_{\tau}^{t}\left|v_{n}(s)\right| d s$. Thus, the sequence $\left\{\theta v_{n}\right\}$ is equicontinuous because the right-hand of the inequality tends to 0 as $t \rightarrow \tau$. Hence, it has a uniformly convergent subsequence by using the Arzela-Ascoli theorem. Choose a subsequence of $\left\{v_{n}\right\}$ (we show it again by $\left\{v_{n}\right\}$ ) such that $\theta v_{n} \rightarrow \theta v$. Hence, $\theta v \in \theta\left(S_{\mathcal{G}, x}\right)$ and so $\mathcal{B}=\theta\left(S_{\mathcal{G}, x}\right)$ is compact for all $x \in S$. Here, we prove that $\mathcal{B} x$ is convex for all $x \in S$. Let $x \in S$ and $u, u^{\prime} \in \mathcal{B} x$. Choose $v, v^{\prime} \in S_{\mathcal{G}, x}$ such that $u(t)=a_{\zeta} v(t)+b_{\zeta} \int_{0}^{t} v(s) d s$ and $u^{\prime}(t)=a_{\zeta} v^{\prime}(t)+b_{\zeta} \int_{0}^{t} v^{\prime}(s) d s$ for almost all $t \in I$. Let $0 \leq \lambda \leq 1$. Then we have

$$
\lambda u(t)+(1-\lambda) u^{\prime}(t)=a_{\zeta}\left(\lambda v(t)+(1-\lambda) v^{\prime}(t)\right)+b_{\zeta} \int_{0}^{t}\left(\lambda v(s)+(1-\lambda) v^{\prime}(s)\right) d s .
$$

Since $\mathcal{G}$ is convex-valued, $\lambda u+(1-\lambda) u^{\prime} \in \mathcal{B} x$. It is clear that $\mathcal{A}$ is bounded, closed, and convex-valued. We show that $\mathcal{A} x \mathcal{B} x$ is a convex subset of $S$ for all $x \in S$. Let $x \in S$ and $u, u^{\prime} \in \mathcal{A x} \mathcal{B} x$. Choose $v, v^{\prime} \in S_{\mathcal{G}, x}$ such that

$$
u(t)=g\left(t, x(t),(\phi x)(t),(\varphi x)(t),{ }_{N}{ }_{N} \mathcal{D}^{\zeta 1} x(t), \ldots,{ }_{N}{ }^{\mathrm{CF}} \mathcal{D}^{\zeta n} x(t)\right) \times\left(a_{\zeta} v(t)+b_{\zeta} \int_{0}^{t} v(s) d s\right),
$$

and

$$
\begin{aligned}
u^{\prime}(t)= & g\left(t, x(t),(\phi x)(t),(\varphi x)(t),{ }_{N}^{\mathrm{CF}} \mathcal{D}^{\zeta 1} x(t), \ldots,{ }_{N}{ }_{N} \mathcal{D}^{\zeta n} x(t)\right) \\
& \times\left(a_{\zeta} v^{\prime}(t)+b_{\zeta} \int_{0}^{t} v^{\prime}(s) d s\right)
\end{aligned}
$$

for almost all $t \in I$. Hence,

$$
\begin{aligned}
\lambda u(t)+(1-\lambda) u^{\prime}(t)= & g(t, x(t),(\phi x)(t),(\varphi x)(t), \\
& \left.{ }_{N}^{\mathrm{CF}} \mathcal{D}^{\zeta_{1}} x(t), \ldots,{ }_{N}^{\mathrm{CF}} \mathcal{D}^{\zeta_{n}} x(t)\right) \\
& \times\left[a_{\zeta}\left(\lambda v(t)+(1-\lambda) v^{\prime}(t)\right)\right. \\
& \left.+b_{\zeta} \int_{0}^{t}\left(\lambda v(s)+(1-\lambda) v^{\prime}(s)\right) d s\right] .
\end{aligned}
$$

Note that $\lambda u+(1-\lambda) u^{\prime} \in \mathcal{A} x \mathcal{B} x$ because $\mathcal{G}$ is convex-valued. Hence, $\mathcal{A} x \mathcal{B} x$ is a convex subset of $\mathcal{X}$ for all $x \in \mathcal{X}$. However, we have

$$
\begin{aligned}
|u(t)| & =\left|g\left(t, x(t),(\phi x)(t),(\varphi x)(t),{ }_{N}^{\mathrm{CF}} \mathcal{D}^{\zeta 1} x(t), \ldots,{ }_{N}^{\mathrm{CF}} \mathcal{D}^{\zeta n} x(t)\right) \times\left(a_{\zeta} v(t)+b_{\zeta} \int_{0}^{t} v(s) d s\right)\right| \\
& \leq K\left(a_{\zeta}+b_{\zeta}\right)\|m\|_{\infty}=L<1
\end{aligned}
$$

for all $t \in I$, and so $u \in S$ and $\mathcal{A} x \mathcal{B} x$ is a convex subset of $S$ for all $x \in S$. Now, we show that the operator $\mathcal{B}$ is compact. It is enough to prove that $\mathcal{B}(S)$ is uniformly bounded and equi-continuous. Let $u \in \mathcal{B}(S)$. Choose $v \in S_{\mathcal{G}, x}$ such that

$$
u(t)=g\left(t, x(t),(\phi x)(t),(\varphi x)(t),{ }_{N}^{\mathrm{CF}} \mathcal{D}^{\zeta_{1}} x(t), \ldots,{ }_{N}^{\mathrm{CF}} \mathcal{D}^{\zeta_{n}} x(t)\right) \times\left(a_{\zeta} v(t)+b_{\zeta} \int_{0}^{t} v(s) d s\right)
$$


for some $x \in S$. Since $|u(t)| \leq K\left(a_{\zeta}+b_{\zeta}\right)\|m\|_{\infty},\|u\|_{\infty}=\max _{t \in I}|u(t)| \leq K\left(a_{\zeta}+b_{\zeta}\right)\|m\|_{\infty}$. Now, we prove that $\mathcal{B}$ maps $S$ to equi-continuous subsets of $\mathcal{X}$. Let $t, \tau \in J$ with $\tau<t, x \in S$, and $u \in \mathcal{B} x$. Choose $v \in S_{\mathcal{G}, x}$ such that $u(t)=a_{\zeta} v(t)+b_{\zeta} \int_{0}^{t} v(s) d s$. Then we have

$$
|u(t)-u(\tau)| \leq a_{\zeta}|v(t)-v(\tau)|+b_{\zeta} \int_{\tau}^{t}|v(s)| d s .
$$

Note that the right-hand side of this inequality tends to 0 as $t \rightarrow \tau$. By using the ArzelaAscoli theorem, we get $\mathcal{B}$ is compact. Here, we show that $\mathcal{B}$ has a closed graph. Let $x_{n} \in S$ and $u_{n} \in \mathcal{B} x_{n}$ for all $n$ with $x_{n} \rightarrow x^{\prime}$ and $u_{n} \rightarrow u^{\prime}$. We show that $u^{\prime} \in \mathcal{B} x^{\prime}$. For each $n$, choose $v_{n} \in S_{\mathcal{G}, x_{n}}$ such that $u_{n}(t)=a_{\zeta} v_{n}(t)+b_{\zeta} \int_{0}^{t} v_{n}(s) d s$ for all $t \in J$. Again, consider the continuous linear operator $\theta: L^{1}(I) \rightarrow \mathcal{X}$ such that $\theta(v)(t)=u(t)=a_{\zeta} v(t)+b_{\zeta} \int_{0}^{t} v(s) d s$. By using Lemma $4, \theta \circ S_{\mathcal{G}}$ is a closed graph operator. Since $x_{n} \rightarrow x^{\prime}$ and $u_{n} \in \theta\left(S_{\mathcal{G}, x_{n}}\right)$ for all $n$, there is $v^{\prime} \in S_{\mathcal{G}, x^{\prime}}$ such that $u^{\prime}(s)=a_{\zeta} v^{\prime}(t)+b_{\zeta} \int_{0}^{t} v^{\prime}(s) d s$. Hence, $u^{\prime} \in \mathcal{B} x^{\prime}$. Thus, $\mathcal{B}$ has a closed graph and so $\mathcal{B}$ is upper semi-continuous. Finally note that

$$
\begin{aligned}
H(\mathcal{A} x, \mathcal{A} y)= & \|\mathcal{A} x-\mathcal{A} y\| \\
= & \max _{t \in I} \mid g\left(t, x(t),(\phi x)(t),(\varphi x)(t),{ }_{N}^{\mathrm{CF}} \mathcal{D}^{\zeta_{1}} x(t), \ldots,{ }_{N}{ }_{\mathrm{CF}} \mathcal{D}^{\zeta_{n}} x(t)\right) \\
& \left.-g\left(t, y(t),(\phi y)(t),(\varphi y)(t),{ }_{N} \mathcal{C F}^{\zeta_{1}} y(t), \ldots,{ }_{N}{ }^{{ }_{N}} \mathcal{D}^{\zeta_{n}} y(t)\right)\right) \mid \\
\leq & \max _{t \in I}|\eta(t)|\left(1+\zeta_{0}+\iota_{0}+\sum_{i=1}^{n} \frac{B\left(\zeta_{i}\right)}{\left(1-\zeta_{i}\right)^{2}}\right)|x(t)-y(t)| \\
= & \eta^{*}\left(\left(1+\zeta_{0}+\iota_{0}+\sum_{i=1}^{n} \frac{B\left(\zeta_{i}\right)}{\left(1-\zeta_{i}\right)^{2}}\right)\|x-y\|_{\infty}\right.
\end{aligned}
$$

for all $x, y \in \mathcal{X}$. Now, by using Theorem 8 , the inclusion problem $x \in \mathcal{A} x \mathcal{B} x$ has a solution which is a solution for problem (4).

In this part, we show that the set of solutions for the second fractional integrodifferential inclusion problem is infinite dimensional under some conditions. First we prove the next result.

Lemma 15 Suppose that $m \in L^{1}\left(I, \mathbb{R}^{+}\right), \mathcal{F}: I \times \mathbb{R}^{m+n+3} \rightarrow \mathcal{P}_{c v, c p}(\mathbb{R})$ is a multivalued map such that the map $t \vdash f\left(t, x_{1}, x_{2}, \ldots, x_{3+m+n}\right)$ is measurable and

$$
\left\|\mathcal{F}\left(t, x_{1}, x_{2}, \ldots, x_{m+n+3}\right)\right\|=\sup \left\{|f|: f \in \mathcal{F}\left(t, x_{1}, x_{2}, \ldots, x_{m+n+3}\right)\right\} \leq m(t)
$$

for almost all $t \in I$ and $\in x_{1}, x_{2}, \ldots, x_{m+n+3} \in \mathbb{R}$. Define $\Phi: \mathcal{X} \rightarrow \mathcal{P}(\mathcal{X})$ by

$$
\Phi(x)=\left\{g \in \mathcal{X}: \text { there is } f \in S_{\mathcal{F}, x} \text { such that } g(t)=a_{\zeta} f(t)+b_{\zeta} \int_{0}^{t} f(s) d \text { s for all } t \in I\right\} .
$$

Then $\Phi(x) \in \mathcal{P}_{\text {cp.cv }}(\mathcal{X})$ for all $x \in \mathcal{X}$.

Proof Note that $\Phi=\theta \circ S_{\mathcal{F}}$, where $\theta: L^{1}(I, \mathbb{R}) \rightarrow \mathcal{X}$ is the continuous linear map defined by $\theta g(t)=a_{\zeta} f(t)+b_{\zeta} \int_{0}^{t} f(s) d s$. Let $x \in \mathcal{X}$ and $\left\{g_{n}\right\}$ be a sequence in $S_{\mathcal{F}, x}$. Then we have

$$
g_{n}(t) \in \mathcal{F}\left(t, x(t),(\phi x)(t),(\psi x)(t),{ }_{N}{ }^{C F} \mathcal{D}^{\beta_{1}} x(t), \ldots,{ }_{N}{ }^{C F} \mathcal{D}^{\beta_{m}} x(t),{ }^{c F} \mathcal{I}^{\gamma_{1}} x(t), \ldots,{ }^{c F} \mathcal{I}^{\gamma_{n}} x(t)\right)
$$


for almost $t \in I$. Since

$$
\mathcal{F}\left(t, x(t),(\phi x)(t),(\psi x)(t),{ }_{N}{ }^{\mathrm{CF}} \mathcal{D}^{\beta_{1}} x(t), \ldots,{ }_{N}{ }^{\mathrm{CF}} \mathcal{D}^{\beta_{m}} x(t),{ }^{c F} \mathcal{I}^{\gamma_{1}} x(t), \ldots,{ }^{c F} \mathcal{I}^{\gamma_{n}} x(t)\right)
$$

is compact for all $t \in I$, there is a convergent subsequence of $\left\{g_{n}(t)\right\}$ (show it by $\left\{g_{n}(t)\right\}$ ) which converges to some $g \in S_{\mathcal{F}, x}$. Note that $\theta g_{n}(t) \rightarrow \theta g(t)$ pointwise on $I$ because $\theta$ is continuous. Here, we prove that $\left\{\theta g_{n}\right\}$ is an equi-continuous sequence. Let $\tau<t \in I$. Then we have $\left|\theta g_{n}(t)-\theta g_{n}(\tau)\right|=a_{\zeta}(f(t)-f(\tau))+b_{\zeta} \int_{\tau}^{t} f(s) d s$. Note that the sequence $\left\{\theta g_{n}\right\}$ is equi-continuous because the right-hand side of the inequality tends to zero when $\tau \rightarrow t$. Thus, there is a uniformly convergent subsequence of $\left\{g_{n}\right\}$ (show it by $\left\{g_{n}\right\}$ again) such that $\theta g_{n} \rightarrow \theta g$ (we use the Arzela-Ascoli theorem). This implies that $\theta g \in \theta\left(S_{\mathcal{F}, x}\right)$. Hence, $\Phi x=\theta\left(S_{\mathcal{F}, x}\right)$ is compact for all $x \in \mathcal{X}$. Now, we show that $\Phi x$ is convex for each $x \in \mathcal{X}$. Let $g, g^{\prime} \in \Phi x$. Choose $f, f^{\prime} \in S_{\mathcal{F}, x}$ such that $\left.g(t)=a_{\zeta} f(t)+b_{\zeta} \int_{0}^{t} f(s) d s\right)$ and $g^{\prime}(t)=a_{\zeta} f^{\prime}(t)+$ $\left.b_{\zeta} \int_{0}^{t} f^{\prime}(s) d s\right)$ for almost all $t \in I$. Let $0 \leq \lambda \leq 1$. Then we have

$$
\lambda g(t)+(1-\lambda) g^{\prime}(t)=a_{\zeta}\left(\lambda f(t)+(1-\lambda) f^{\prime}(t)\right)+b_{\zeta} \int_{0}^{t}\left(\lambda f(s)+(1-\lambda) f^{\prime}(s)\right) d s .
$$

Since $S_{\mathcal{F}, x}$ is convex, $\lambda g+(1-\lambda) g^{\prime} \in \Phi x$. This completes the proof.

Note that the fixed point set of $\Phi$ is equal to the set of solutions for the inclusion problem (2). Now by using some different conditions, we show that the set of solutions for the fractional integro-differential inclusion problem could be infinite dimensional.

Theorem 16 Suppose that $\eta \in L^{1}\left(I, \mathbb{R}^{+}\right), \mathcal{F}: I \times \mathbb{R}^{m+n+3} \rightarrow \mathcal{P}_{c v, c p}(\mathbb{R})$ is a multivalued map such that the function $t \vdash \mathcal{F}\left(t, x_{1}, x_{2}, \ldots, x_{m+n+3}\right)$ is measurable,

$$
H\left(\mathcal{F}\left(t, x_{1}, x_{2}, \ldots, x_{m+n+3}\right), \mathcal{F}\left(t, y_{1}, y_{2}, \ldots, y_{m+n+3}\right)\right) \leq \eta(t) \sum_{i=1}^{m+n+3}\left|x_{i}-y_{i}\right|
$$

and $\left\|\mathcal{F}\left(t, x_{1}, x_{2}, \ldots, x_{m+n+3}\right)\right\|=\sup \left\{|f|: f \in \mathcal{F}\left(t, x_{1}, x_{2}, \ldots, x_{m+n+3}\right)\right\} \leq \eta(t)$ for almost all $t \in$ $I$ and $\in x_{1}, x_{2}, \ldots, x_{m+n+3}, y_{1}, y_{2}, y_{m+n+3} \in \mathbb{R}$. If Lebesgue measure of the set

$$
\left\{t: \operatorname{dim} \mathcal{F}\left(t, x_{1}, x_{2}, \ldots, x_{m+n+3}\right)<1 \text { for some } x_{1}, x_{2}, \ldots, x_{m+n+3} \in \mathbb{R}\right\}
$$

is zero and $\Delta<1$, then the set of all solutions for problem (2) is infinite dimensional, where $\Delta=\eta^{*}\left(1+n+\zeta_{0}+\iota_{0}+\sum_{i=1}^{m} \frac{B\left(\beta_{i}\right)}{\left(1-\beta_{i}\right)^{2}}\right)\left(1+n+\sum_{i=1}^{m} \frac{B\left(\beta_{i}\right)}{\left(1-\beta_{i}\right)^{2}}\right)$.

Proof Similar to Lemma 15, define the multivalued map $\Phi: \mathcal{X} \rightarrow \mathcal{P}(\mathcal{X})$ by

$$
\Phi(x)=\left\{g \in \mathcal{X}: \text { there is } f \in S_{\mathcal{F}, x} \text { such that } g(t)=a_{\zeta} f(t)+b_{\zeta} \int_{0}^{t} f(s) d s \text { for all } t \in I\right\} .
$$

By using Lemma $15, \Phi x \in \mathcal{P}_{c p, c v}(\mathcal{X})$ for all $x \in \mathcal{X}$. By using a similar proof in Theorem 12, we can prove that $\Phi$ is a contractive multivalued map. Now, we show that $\operatorname{dim} \Phi x>k$ for all $x \in \mathcal{X}$ and $k \geq 1$. Let $k \geq 1, x \in \mathcal{X}$, and

$$
\begin{aligned}
\mathcal{G}(t)= & \mathcal{F}\left(t, x(t),(\phi x)(t),(\psi x)(t),{ }_{N}{ }^{\mathrm{CF}} \mathcal{D}^{\beta_{1}} x(t),{ }_{N}{ }_{N} \mathcal{D}^{\beta_{2}} x(t), \ldots,{ }_{N}{ }^{\mathrm{CF}} \mathcal{D}^{\beta_{m}} x(t),\right. \\
& \left.{ }^{c F} \mathcal{I}^{\gamma_{1}} x(t),{ }^{c F} \mathcal{I}^{\gamma_{2}} x(t), \ldots,{ }^{c F} \mathcal{I}^{\gamma_{n}} x(t)\right)
\end{aligned}
$$


for all $t \in I$. By using Lemma 9, there are linearly independent measurable selections $g_{1}, \ldots, g_{k}$ for $\mathcal{G}$. Consider the maps $h_{i}(t)=a_{\zeta} g_{i}(t)+b_{\zeta}(t) \int_{0}^{t} g_{i}(s) d s$ for $i=1, \ldots, k$. Assume that $\sum_{i=1}^{k} a_{i} h_{i}(t)=0$ for almost $t \in I$. Since $a_{\zeta}, b_{\zeta} \neq 0$, by using the Caputo-Fabrizio derivatives, we get $\sum_{i=1}^{k} a_{i} g_{i}(t)=0$ for almost $t \in I$. Hence, $a_{1}=\cdots=a_{k}=0$. This implies that $h_{1}, \ldots, h_{k}$ are linearly independent, and so $\operatorname{dim} \Phi x \geq k$. Hence, we conclude that the set of fixed points of $\Phi$ is infinite dimensional by using Theorem 10 . Thus, the set of all solutions for problem (2) is infinite dimensional.

\section{Conclusion}

We guess that researchers will review different more fractional integro-differential inclusions in the near future. In this manuscript, we first investigate the existence of solutions for four fractional integro-differential inclusions including the new Caputo-Fabrizio derivation which has been introduced recently. Also, we show that dimension of the set of solutions for the second fractional integro-differential inclusion problem is infinite dimensional under some different conditions.

\section{Acknowledgements}

The second and third authors were supported by Azarbaijan Shahid Madani University. The authors express their gratitude to the unknown referees for their helpful suggestions which improved the final version of this paper.

\section{Funding}

Not applicable.

Availability of data and materials

Data sharing not applicable to this paper as no datasets were generated or analyzed during the current study.

\section{Competing interests}

The authors declare that they have no competing interests.

\section{Authors' contributions}

Each of the authors contributed to each part of this study equally and approved the final version of the manuscript.

\section{Author details}

${ }^{1}$ Department of Mathematics, Cankaya University, Ankara, Turkey. ${ }^{2}$ Institute of Space Sciences, Magurele-Bucharest, Romania. ${ }^{3}$ Department of Mathematics, Azarbaijan Shahid Madani University, Tabriz, Iran.

\section{Publisher's Note}

Springer Nature remains neutral with regard to jurisdictional claims in published maps and institutional affiliations.

Received: 16 October 2018 Accepted: 10 April 2019 Published online: 24 April 2019

References

1. Agarwal, R.P., Baleanu, D., Hedayati, V., Rezapour, S.: Two fractional derivative inclusion problems via integral boundary condition. Appl. Math. Comput. 257, 205-212 (2015)

2. Atangana, A.: Non validity of index law in fractional calculus: a fractional differential operator with Markovian and non-Markovian properties. Phys. A, Stat. Mech. Appl. 505, 688-706 (2018)

3. Atangana, A.: Blind in a commutative world: simple illustrations with functions and chaotic attractors. Chaos Solitons Fractals 114, 347-363 (2018)

4. Atangana, A., Gómez-Aguilar, J.F.: Hyper-chaotic behavior obtained via a nonlocal operator with exponential decay and Mittag-Leffler laws. Chaos Solitons Fractals 102, 285-294 (2017)

5. Atangana, A., Gómez-Aguilar, J.F.: Decolonisation of fractional calculus rules: breaking commutativity and associativity to capture more natural phenomena. Eur. Phys. J. Plus 133, 166 (2018)

6. Atangana, A., Gómez-Aguilar, J.F.: A new derivative with normal distribution kernel: theory, methods and applications. Phys. A, Stat. Mech. Appl. 476, 1-14 (2017)

7. Atangana, A., Gómez-Aguilar, J.F.: Fractional derivatives with no-index law property: application to chaos and statistics. Chaos Solitons Fractals 114, 516-535 (2018)

8. Baleanu, D., Agarwal, R.P., Mohammadi, H., Rezapour, S.: Some existence results for a nonlinear fractional differential equation on partially ordered Banach spaces. Bound. Value Probl. 2013, 112 (2013)

9. Baleanu, D., Rezapour, S., Mohammadi, H.: Some existence results on nonlinear fractional differential equations. Philos. Trans. R. Soc., Math. Phys. Eng. Sci. 371, 20120144 (2013)

10. Baleanu, D., Mousalou, A., Rezapour, S.: A new method for investigating approximate solutions of some fractional integro-differential equations involving the Caputo-Fabrizio derivative. Adv. Differ. Equ. 2017(1), 51 (2017) 
11. Baleanu, D., Mousalou, A., Rezapour, S.: The extended fractional Caputo-Fabrizio derivative of order $0 \leq \sigma<1$ on $c_{\mathbb{R}}[0,1]$ and the existence of solutions for two higher-order series-type differential equations. Adv. Differ. Equ. 2018(1), 255 (2018)

12. Etemad, S., Rezapour, S.: On a two-variables fractional partial differential inclusion via Riemann-Liouville derivative. Novi Sad J. Math. 46(2), 45-53 (2016)

13. Etemad, S., Rezapour, S.: On a system of hyperbolic partial fractional differential inclusions. Novi Sad J. Math. 46(2) 145-161 (2016)

14. Gómez-Aguilar, J.F., Atangana, A.: New insight in fractional differentiation: power, exponential decay and Mittag-Leffler laws and applications. Eur. Phys. J. Plus 132, 13 (2017)

15. Rezapour, S., Hedayati, V.: On a Caputo fractional differential inclusion with integral boundary condition for convex-compact and nonconvex-compact valued multifunctions. Kragujev. J. Math. 41(1), 143-158 (2017)

16. Shabibi, M., Rezapour, S., Vaezpour, S.M.: A singular fractional integro-differential equation. Sci. Bull. "Politeh." Univ. Buchar., Ser. A, Appl. Math. Phys. 79(1), 109-118 (2017)

17. Shabibi, M., Postolache, M., Rezapour, S.: Positive solutions for a singular sum fractional differential system. Int. J. Anal. Appl. 13(1), 108-118 (2017)

18. Tateishi, A.A., Ribeiro, H.V., Lenzi, E.K.: The role of fractional time-derivative operators on anomalous diffusion. Front. Phys. 25, 52 (2017)

19. Caputo, M., Fabrizzio, M.: A new definition of fractional derivative without singular kernel. Prog. Fract. Differ. Appl. 1(2), 73-85 (2015)

20. Alsaedi, A., Baleanu, D., Etemad, S., Rezapour, S.: On coupled systems of time-fractional differential problems by using a new fractional derivative. J. Funct. Spaces 2016, 4626940 (2016)

21. Algahtani, O., Jefain, J: Numerical approximation of the space-time Caputo-Fabrizio fractional derivative and application to groundwater pollution equation. Chaos Solitons Fractals 89, 552-559 (2016)

22. Atangana, A., Alqahtani, R.T.: Numerical approximation of the space-time Caputo-Fabrizio fractional derivative and application to groundwater pollution equation. Adv. Differ. Equ. 2016, 156 (2016)

23. Gómez-Aguilar, J.F., Yépez-Martínez, H., Calderón-Ramón, C., Cruz-Orduña, I., Escobar-Jiménez, R.F., Olivares-Peregrino, V.H.: Modeling of a mass-spring-damper system by fractional derivatives with and without a singular kernel. Entropy 17(9), 6289-6303 (2015)

24. Goufo, E.F., Doungmo, P., Morgan, K., Mwambakana, J.N.: Duplication in a model of rock fracture with fractional derivative without singular kernel. Open Math. 13, 839-846 (2015)

25. Kaczorek, T., Borawski, K.: Fractional descriptor continuous-time linear systems described by the Caputo-Fabrizio derivative. Int. J. Appl. Math. Comput. Sci. 26(3), 533-541 (2016)

26. Agarwal, R.P., Ahmad, B., Alsaedi, A., Shahzad, N.: Dimension of the solution set for fractional differential inclusions, J. Nonlinear Convex Anal. 14(2), 319-329 (2013)

27. Losada, J., Nieto, J.J.: Properties of a new fractional derivative without singular kernel. Prog. Fract. Differ. Appl. 1(2), 87-92 (2015)

28. Lasota, A., Opial, Z: An application of the Kakutani-Ky Fan theorem in the theory of ordinary differential equations. Bull. Acad. Pol. Sci., Sér. Sci. Math. Astron. Phys. 13, 781-786 (1965)

29. Granas, A., Dugundji, J.: Fixed Point Theory. Springer, Berlin (2005)

30. Covitz, H., Nadler, S.: Multi-valued contraction mappings in generalized metric spaces. Isr. J. Math. 8(1), 5-11 (1970)

31. Petrusel, A.: Fixed point and selections for multi-valued operators. Fixed Point Theory 2, 3-22 (2001)

32. Dhage, B.C.: Multi-valued operators and fixed point theorems in Banach algebras. Taiwan. J. Math. 10(4), 1024-1045 (2006)

33. Kamenskii, M., Obukhovskii, V., Zecca, P.: Condensing Multi-Valued Maps and Semilinear Differential Inclusions in Banach Spaces. De Gruyter, Berlin (2001)

34. Castaing, C., Valadier, M.: A New Definition of Fractional Derivative Without Singular Kernel. Springer, Berlin (1977)

\section{Submit your manuscript to a SpringerOpen ${ }^{\circ}$ journal and benefit from:}

- Convenient online submission

- Rigorous peer review

- Open access: articles freely available online

- High visibility within the field

- Retaining the copyright to your article

Submit your next manuscript at $\boldsymbol{\nabla}$ springeropen.com 